\title{
5G Multi-Carrier Modulation Techniques: Prototype Filters, Power Spectral Density, and Bit Error Rate Performance
}

\section{Shaik Nilofer ( $\sim$ nilofershaik@gmail.com )}

Lovely Professional University Faculty of Technology and Sciences https://orcid.org/0000-0001-80697319

Praveen Kumar Malik

Lovely Professional University Faculty of Technology and Sciences

\section{Research Article}

Keywords: 5G Air Interface, Candidate Waveforms, FBMC, F-OFDM, GFDM, OFDM, Power spectral density, UFMC

Posted Date: March 24th, 2021

DOI: https://doi.org/10.21203/rs.3.rs-345216/v1

License: (c) (i) This work is licensed under a Creative Commons Attribution 4.0 International License. Read Full License 


\title{
5G Multi-Carrier Modulation Techniques: Prototype Filters, Power Spectral Density, and Bit Error Rate Performance
}

\author{
Nilofer Shaik ${ }^{1}$, Praveen Kumar Malik ${ }^{2}$ \\ 1nilofershaik@gmail.com, 2pkmalikmeerut@gmail.com \\ ${ }^{1-2}$ Lovely Professional University, Phagwara, Punjab, India \\ Corresponding author: Nilofer Shaik, nilofershaik@gmail.com
}

\begin{abstract}
In recent years for fourth-generation wireless communication systems Orthogonal Frequency Division Multiplexing (OFDM) multi-carrier modulation (MCM) has been an excellent choice. But for the fifth-generation air interface, the choice of multi-carrier modulation remains the biggest challenge. A potential multi-carrier modulation or candidate waveforms are required for the design of the physical layer. This paper highlights the merits and limitations of new candidate waveforms for fifth-generation wireless communication system. This paper mainly reviews Orthogonal frequency division multiplexing, Filtered-OFDM (F-OFDM), Universal Frequency Division Multiplexing (UFMC), Filtered Bank Multi-Carrier Modulation (FBMC), and Generalized Frequency Division Multiplexing (GFDM). This paper mainly reviews some of the comparisons of the parameters as power spectral density and bit error rate.
\end{abstract}

Keywords: 5G Air Interface, Candidate Waveforms, FBMC, F-OFDM, GFDM, OFDM, Power spectral density, UFMC.

1. Introduction: In modern years the research community is mainly working on the $5 \mathrm{G}$ wireless communication system (WCS) requirements which provide immense data rate, small latency, less energy consumption, immense scalability, increase in connectivity, reliability, and to have higher security. The architecture of $5 \mathrm{G}$ wireless communication system which is been modeled these years is facing these challenges are needed to be designed. The density of base stations is increased which will achieve a high degree of network capacity. It also provides a high level of frequency reuse [1] using femto and pico cells. Simplicity is the main objective of 5G WCS architecture. An alternate form has to be adopted as it is expected rapid growth in the number of connections and reducing the complexity as well as the cost of the system architecture. Massive MIMO (mMIMO) systems, massive machine-type communications, enhanced mobile broadband, and ultra-reliable low latency communications are the main types of communications that have become an important requirement for 5G WCS [2-4]. From the perspective of the physical layer, an efficient spectrum slicing plays a major role to support several numbers of requirements in 5G. So as a key challenge a new candidate waveform is required in 5G WCS [5-6]. LTE and LTE-A had adapted a profound procedure for the candidate waveform in terms of cyclic prefix OFDM (CP-OFDM). As there is an increase in demand for the newer applications which has to provide high bandwidth, connecting several numbers of devices, low latency, high spectral efficiency, and higher data rates has influenced the choice of new candidate waveforms that fulfill the above needs [7]. This paper mainly focuses on the new candidate waveforms such as F-OFDM [8], FBMC [9-11], UFMC [12-13], and GFDM [14-15] in comparison with CPOFDM. 
2. Candidate waveforms system model for 5G: This section deals with some of the new candidate waveforms that are promised as an efficient multi-carrier modulation technique for $5 \mathrm{G}$. The design of multicarrier modulation techniques is depended on the tendency of spectral containment, constraint on hardware, and propagation channel [16]. The problems that are identified using previous multi-carrier modulation techniques are inclined in Table 1.

Table 1: Drawbacks of existing MCM techniques.

\begin{tabular}{|l|l|}
\hline \multicolumn{1}{|c|}{ Problem Parameters } & \multicolumn{1}{c|}{ Reasons } \\
\hline Design Complexity & To suit 5G applications which require complex transceiver. \\
\hline Robustness & Work against hardware and software impairments. \\
\hline Spectral Efficiency & Wider bandwidth has to be divided into smaller bandwidth which provides higher data rates. \\
\hline Time Localization & It enables it to efficiently use TDD with low latency. \\
\hline Scalability and Flexibility & $\begin{array}{l}\text { To achieve localization signals both in frequency and time domain and to support } \\
\text { asynchronous requirements. }\end{array}$ \\
\hline PAPR & $\begin{array}{l}\text { To provide robustness over frequency and time channel selectivity and to attain large } \\
\text { frequencies using power amplifiers. }\end{array}$ \\
\hline
\end{tabular}

To attain high data rates, high spectral efficacy, low latency, increasing battery life by reducing PAPR and mMIMO compatibility [17] a new candidate waveform is required for the design of the physical layer in 5G systems architecture. The waveform modulator block diagram is shown in figure 1.

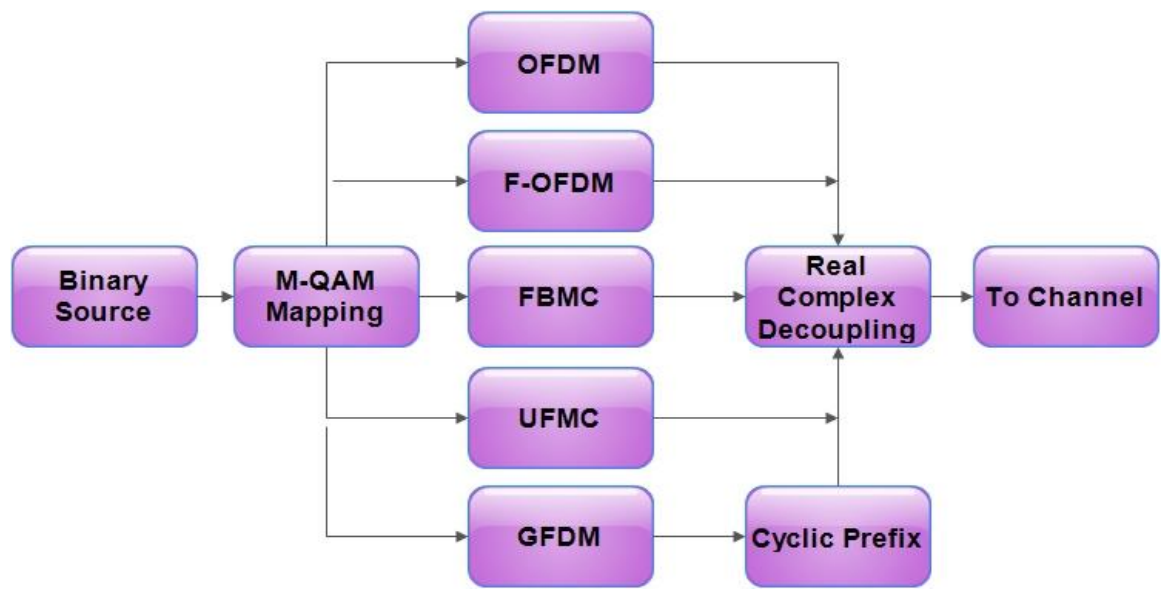

Figure 1: Waveform modulator block diagram.

The information is sent in terms of pulses that overlap in frequency and time for multi-carrier systems. This becomes a great advantage as the pulses utilize a low bandwidth. The broadband channels which are frequency selective transform into multiple subchannels that are frequency flat and have less or zero interference. This enhances the simple equalizers that uses a single tap and is related in symbol detection using maximum likelihood (ML) in a situation of gaussian noise. This also simplifies the procedure of estimating the channel, provides adaptive coding and modulation procedures that are employed in mMIMO [18]. The mathematical expression for the sent signal of a multi-carrier system in the time domain is given as

$$
s(t)=\sum_{k=0}^{K-1} \sum_{l=0}^{L-1} g_{l, k}(t) x_{l, k}
$$


Where $x_{l, k}=$ sent symbol at subcarrier position $l$ and time position $k$. It is selected from a symbol alphabet which a QAM. The transmitted basis pulse is given as

$$
g_{l, k}(t)=p(t-k T) e^{j 2 \pi l F(t-K T)} e^{j \theta_{l, k}}
$$

Where $\mathrm{p}(\mathrm{t})=$ Prototype filter with frequency and time-shifted version

$\mathrm{T}=$ Time spacing

$\mathrm{F}=$ Frequency spacing or Subcarrier spacing.

As the signal is sent over a channel the obtained symbols are decoded by projecting the obtained signal onto basis pulses and is given as

$$
y_{l, k}=\int_{-\infty}^{\infty} r(t) g_{l, k}^{*}(t) d t
$$

It maximizes the SNR in an AWGN channel. The basis pulses are chosen differently at the transceiver based on the requirement. The multi-carrier system has to fulfill some of the important properties as given below:

i. $\quad$ Density of a symbol (Maximum) $T F=1$

ii. Localization on time $\sigma_{t}<\infty$ and Localization on frequency $\sigma_{f}<\infty$

iii. Orthogonality

At least one of these required characteristics has to be compromised while designing the candidate waveforms system had been stated using Balian-Low theorem.

\subsection{Cyclic Prefix Orthogonal Frequency Division Multiplexing (CP-OFDM) System Model:}

CP-OFDM is mostly used candidate waveform in LTE and LTE-A [19]. To form complex symbols the digital data input is mapped using M-QAM or M-PSK modulation schemes. These complex symbols are mapped as orthogonal subcarriers by using IFFT.CP is added to send symbols to avoid interference and give robustness to the transferred signals. The transceiver model of CP-OFDM is shown in figure 2. CP is chosen to have a time duration larger than the delay spread of the channel between the transmitter and receiver end.

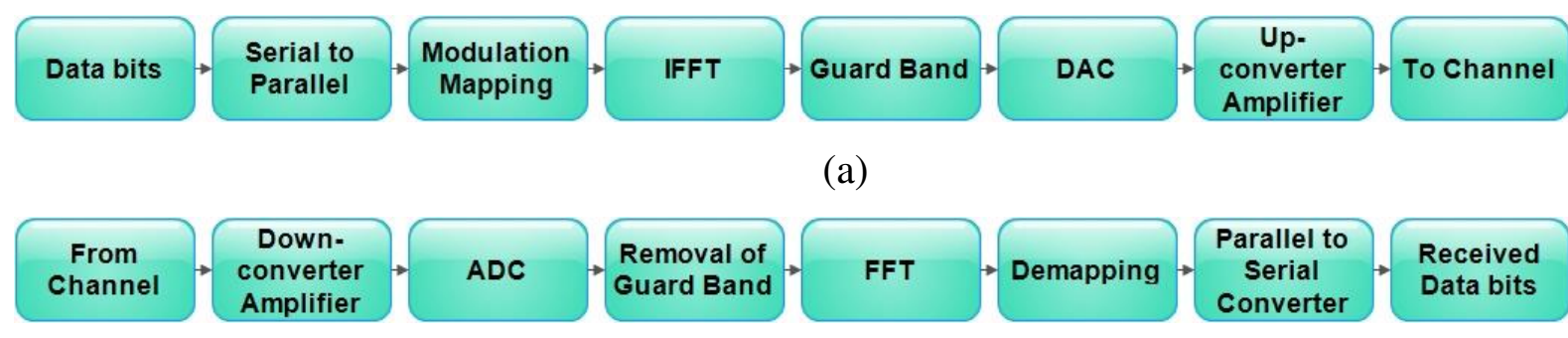

(b)

Figure 2. CP-OFDM transceiver block diagram a. Transmitter b. Receiver

To reduce the computational complexity, CP-OFDM uses rectangular transmitter and receiver pulses. The transmitted pulse is little more than the receiver pulse. This is used to maintain orthogonality in channels thar are selected in frequency. The transmitter prototype filter $(\mathrm{PF})$ is given as

$$
P_{T x}(t)=\left\{\begin{array}{l}
\frac{1}{\sqrt{T_{0}}} \text { if }-\left(\frac{T_{0}}{2}+T_{c p}\right) \leq t<\frac{T_{0}}{2} \\
0 \quad \text { Otherwise }
\end{array}\right.
$$


The receiver $\mathrm{PF}$ is given as

$$
P_{R x}(t)=\left\{\begin{array}{cr}
\frac{1}{\sqrt{T_{0}}} & \text { if }-\frac{T_{0}}{2} \leq t<\frac{T_{0}}{2} \\
0 & \text { Otherwise }
\end{array}\right.
$$

Where $\mathrm{T}_{0}=$ Time Spacing

The main disadvantage of CP-OFDM is as it has no control over OOBE due to rectangular pulse. The other limitations of CP-OFDM are high PAPR and transmission overhead due to CP insertion.

2.2 Filtered-OFDM (F-OFDM) System Model: To enhance the network data rate a larger bandwidth has to be assigned for the $5 \mathrm{G}$ systems [20]. For 5G networks, F-OFDM is considered as one of the efficient candidate waveforms [21-22]. The F-OFDM transceiver is shown in figure 3.

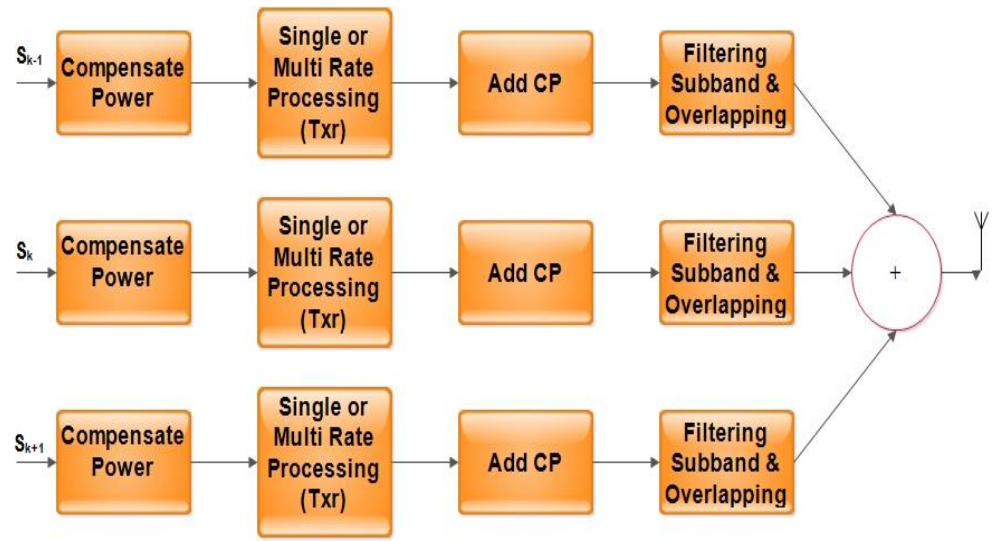

(a)

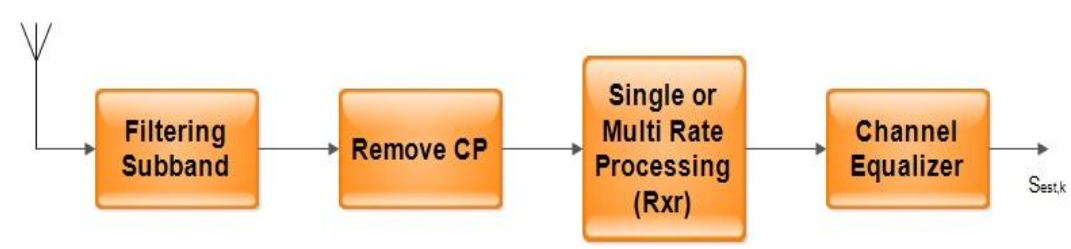

(b)

Figure 3: Transceiver of F-OFDM system model a. Transmitter b. Receiver.

The F-OFDM uses a low dimension IDFT of full size. An N-point IDFT/DFT processed for mapping the signal with a sub-band by using respective columns. The number of subcarriers in one subband will have the same size as IDFT. The complete baseband bandwidth is spread across the signal. The up sampling divides the signal into $1 / \mathrm{K}$ of the full bandwidth. It executes (K-1) image signals based on the sampling theorem. It also creates subband filter of an anti-image which are used to eradicate the signals that contain image. One of the limitations of F-OFDM system is the use of non-ideal filters which causes a reduction in the performance of the system [23]. Because of using low dimension DFT there will be a reduction in system complexity and the filtering operation also helps in reducing the complexity by using the up-sampling procedure. By keeping a long filter length, the OOBE and interference reduce. In uplink transmission, the DFT spreading is employed to reduce PAPR. The obtained signal by using up and down sampling of the k-th subband is given as 


$$
y_{k}=\sum_{l=1}^{K} \frac{1}{\rho_{l}} V^{H} U^{H} T C_{k} B_{l} A_{l} R U V E_{l} s_{l}+\widetilde{v_{k}}
$$

Where V= M-point matrix of IDFT (Normalized)

$\mathrm{U}=$ Matrix with factor $\mathrm{K}$ (Up sampling)

$\mathrm{v}=$ Noise matrix.

The limitation of F-OFDM is higher complexity compared to OFDM but it supports multiple users or services. In F-OFDM systems of single rate the sampling rate is kept same for all subbands and is fixed. In F-OFDM systems of multi-rate, the sampling rate is variable and it uses low complexity low dimension DFT. So that making F-OFDM suitable for 5G systems.

2.3 Filter Bank Multi-Carrier (FBMC) System Model: FBMC has obtained a potential gain in 5G networks compared to OFDM limitations. The main criteria of usage are high spectral efficiency and strict synchronization constraints. The transceiver of FBMC is shown in figure 4.

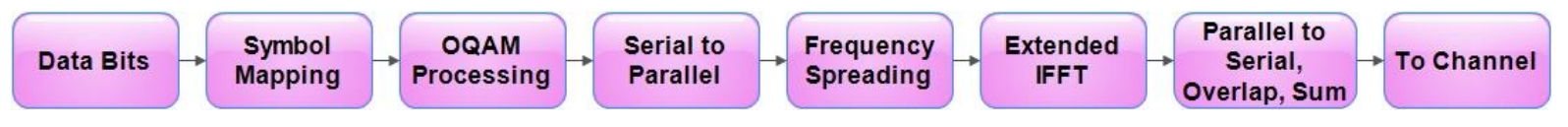

(a)

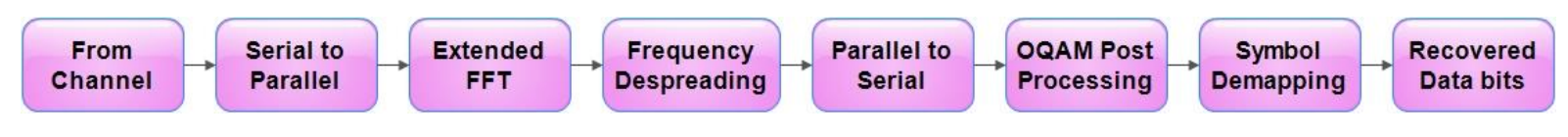

(b)

Figure 4: Transceiver of FBMC a. Transmitter b. Receiver.

Just using a small modification in general OFDM system FBMC system is designed. Each of the different subcarriers is filtered to reduce side lobes which reduce interference which in turn reduces the OOBE. There is no need of adding CP so increases in achievement of attaining high data rates. FBMC and OFDM has the same symbol density but $C P$ is not used. It works with a principle of first designing a PF with $p(t)=p(-t)$. It is orthogonal in nature for frequency and time spacing where, Time spacing is given as $T=T_{o}$ and Frequency spacing is given as $\mathrm{F}=2 / \mathrm{T}_{\mathrm{o}}$. A Hermite $\mathrm{PF}$ for FBMC is given as

$$
p(t)=\frac{1}{\sqrt{T_{0}}} e^{-2 \pi\left(\frac{t}{T_{o}}\right)^{2}} \sum_{\substack{t=\{0,4,8,12, 16,20\}}} \alpha_{i} H_{i}\left(2 \sqrt{\pi} \frac{t}{T_{0}}\right) .
$$

The PF is proposed based on Hermite polynomials $\mathrm{H}_{n}($.$) [24] and its coefficients \mathrm{a}_{\mathrm{i}}$ are can be referred from [25]. There is another type of PF used for FBMC that is PHYDYAS PF [26] and is given as

$$
p(t)= \begin{cases}\frac{1+2 \sum_{i=1}^{O-1} b_{i} \cos \left(\frac{2 \pi t}{O T_{0}}\right)}{O \sqrt{T_{0}}} & \text { if }-\frac{O T_{0}}{2}<t<\frac{O T_{0}}{2} . \\ 0 & \text { Otherwise }\end{cases}
$$

The coefficients of $b_{i}$ are referred from [27]. The second principle of FBMC is to reduce orthogonal by a factor of two for time spacing that is $\mathrm{T}=\mathrm{T}_{0} / 2$ and $\mathrm{F}=1 / \mathrm{T}_{0}$. The last principle is of shifting the induced interference to purely imaginary domain and the phase shift is given as

$$
\theta_{l, k}=\frac{\pi}{2}(l+k)
$$


Loss of complex orthogonality is the limitation in FBMC. The Hermite PF and PHYDYAS PF filter with their time domain and frequency domain spacing representation are shown in figure 5.

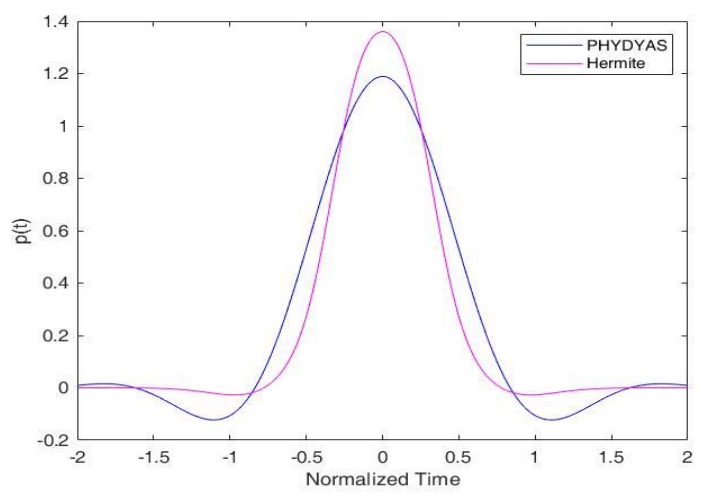

(a)

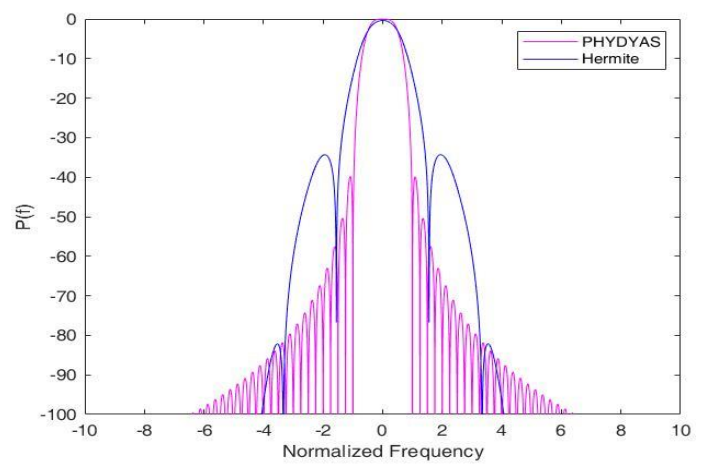

(b)

Figure 5: Prototype Filter for FBMC a. Time Domain Spacing b. Frequency Domain Spacing

The spectral efficiency of the Hermite filter and PHYDYAS filter is shown in figure 6. The spectral efficiency of the Hermite filter is good when subcarrier spacing is taken a normal value, if the subcarrier spacing value is considered infinite then PHYDYAS filter spectral efficiency is good compared to the Hermite filter.

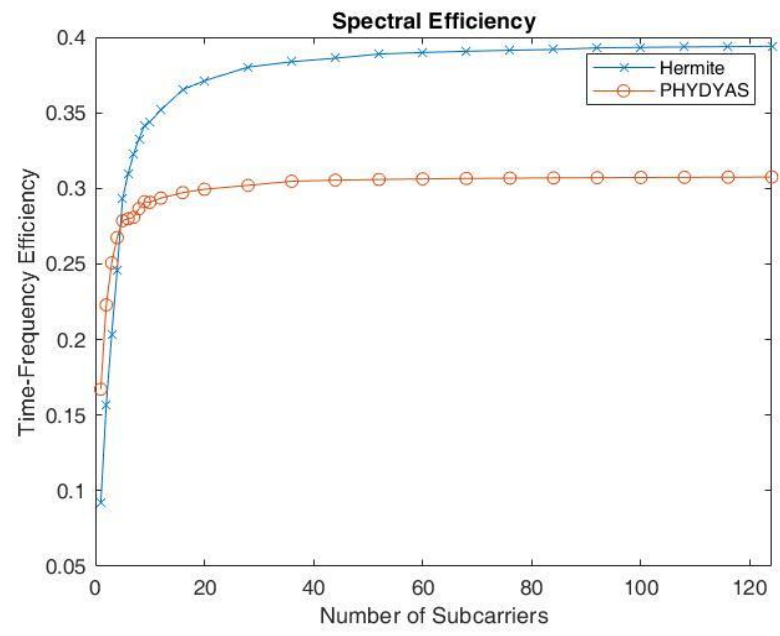

(a) 


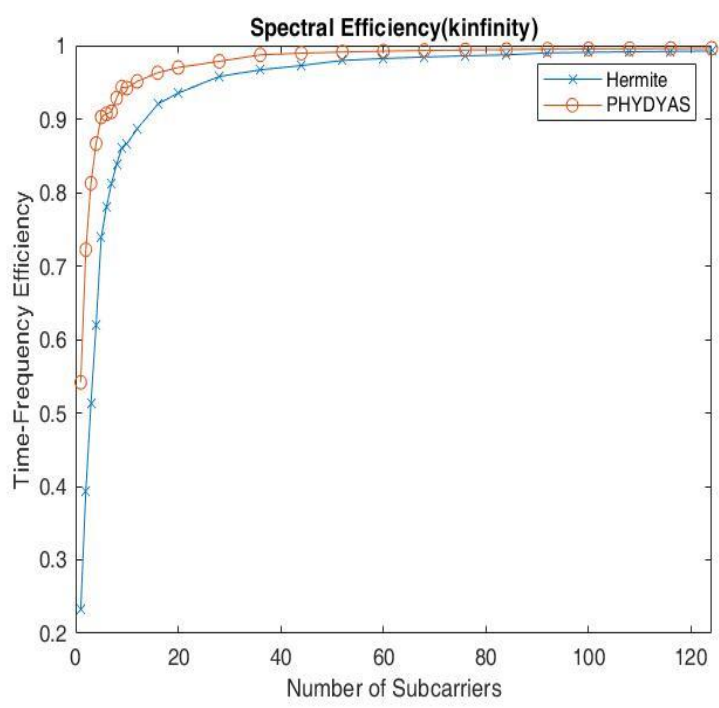

(b)

Figure 6: Spectral efficiency of Hermite and PHYDYAS Filter a. K= minimum b. K= Infinite

2.4 Universal Filtered Multicarrier (UFMC) System Model: It is a candidate waveform that employs subcarrier filtering. The efficacy of UFMC is good in comparison to OFDM in terms of BER and sidelobe attenuation [28]. UFMC is candidate waveform is also the same as F-OFDM and FBMC candidate waveform. In UFMC a group of subcarrier modulation is done. The length of the filter and performance time is reduced based on the grouping of subcarrier. The transceiver of UFMC is shown in figure 7.

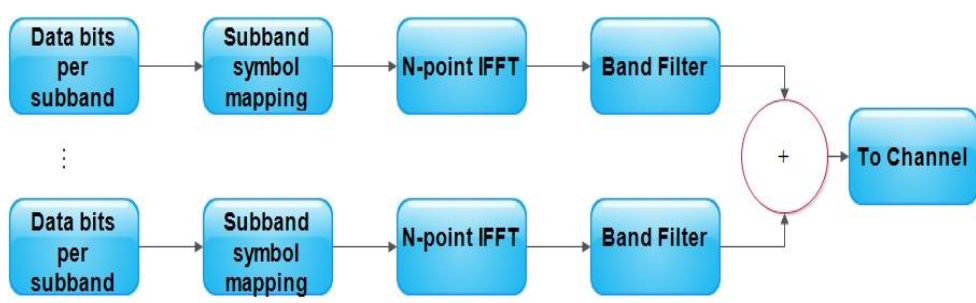

(a)

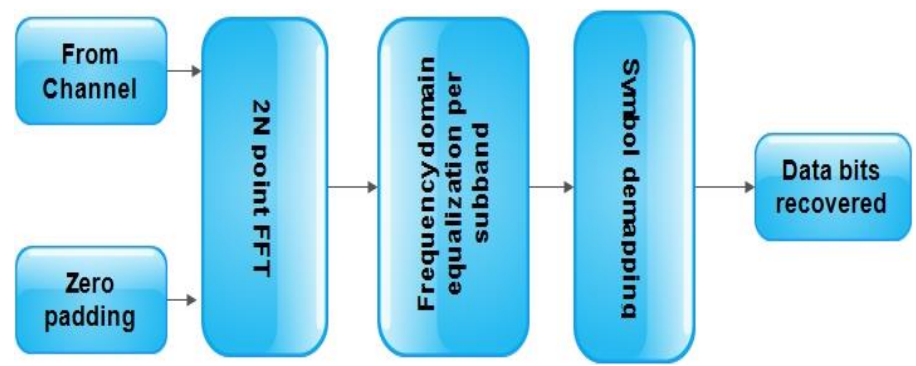

(b)

Figure 7: Transceiver of UFMC a. Transmitter b. Receiver.

This multi-carrier modulation sub-bands are obtained by dividing full band and modulation is performed on individual sub-bands which consists of subcarriers which are fixed in number. The closely spaced individual and narrowed sub-bands go through N-point IFFT to obtain each sub-band in terms of time domain. After the $\mathrm{N}$-point IFFT, the output is given as 


$$
Y_{i}=\operatorname{IFFT}\left\{x_{i}\right\}
$$

The output of the signal after filtered by length $\mathrm{L}$ is given as

$$
y=H \cdot \sim Q \cdot y_{i}
$$

Where $\mathrm{H}=$ Toeplitz matrix with dimensions $(\mathrm{N}+\mathrm{L}-1) * \mathrm{~N}$

$\sim Q=$ Inverse Fourier matrix.

UFMC receiver performs $2 \mathrm{~N}$ point FFT on data received from the channel. To prevent ISI because of transmitter filter, delay a guard of interval zeros is appended between successive IFFT symbols and is given as

$$
\sim Y=F F T\left\{\left|y^{T}, 0,0,0 \ldots \ldots . .0\right|\right\}
$$

The received signal $\mathrm{Y}$ has $\mathrm{N}$ length-frequency domain odd subcarrier points by eliminating even subcarrier points. To detect the sent signal equalization is performed and symbol de-mapping is performed before equalization to the exact data bits.

2.5 Generalized Frequency Division Multiplexing (GFDM) System Model: GFDM is nonorthogonal in nature [29]. It has become one of the promising multi-carrier modulations for $5 \mathrm{G}$ systems. The main advantage of this GFDM is it provides low latency, low PAPR, low OOBE, and low adjacent channel leakage ratio. It has a flexible transceiver design. It provides a high degree of freedom for transceiver design which makes it to be useful in many types of applications such as cognitive radio and full-duplex radio. GFDM based cognitive radio and full-duplex radio are good answers for spectrum management in $5 \mathrm{G}$ systems. The transceiver of GFDM is shown in figure 8 .

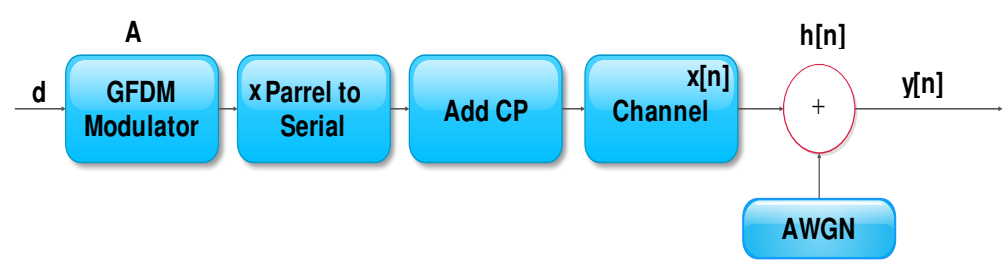

(a)

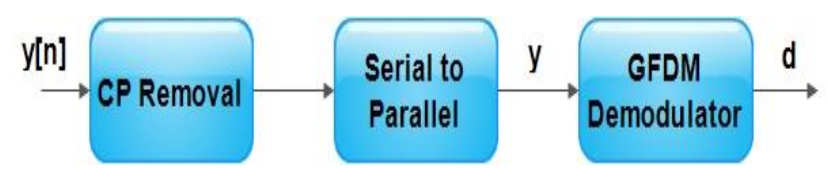

(b)

Figure 8: Transceiver of GFDM a. Transmitter b. Receiver

GFDM is a block-based candidate waveform [30]. It has $\mathrm{K}$ subcarriers with every transmitted $\mathrm{M}$ complexvalued sub symbols. The total number of transmitted signals within the block is given as

$$
\mathrm{D}=\mathrm{KM} \text {. }
$$

The pulse shaped vector for the GFDM block is given as

$$
\left[g_{k, m}\right]_{n}=[g]_{\langle n-m K\rangle D} e^{j 2 \pi k n / K}
$$

With the $\mathrm{m}^{\text {th }}$ symbol on the $\mathrm{k}^{\text {th }}$ subcarrier. 
The prototype of the filter is given as

$$
g \in C^{D}
$$

The transmitter sample vector is given as

$$
\mathrm{x}=\operatorname{Ad}
$$

Where $\mathrm{A}=$ Transmitter matrix

$\mathrm{d}=$ GFDM block for $d \in \mathrm{C}^{D}$

The transmitter sample vector is given as

$$
[x]_{n}=\sum_{k=0}^{K-1} \sum_{m=0}^{M-1}[d]_{k+m K}[g]_{\langle n-m K\rangle D} e^{j 2 \pi k n / K}
$$

Parallel to serial conversion is performed after transmitting $\mathrm{x}$ and $\mathrm{CP}$ is added with a length $\mathrm{L}$ which generates GFDM digital baseband transmit signal. The baseband receiver output is given as

$$
\mathrm{Y}[\mathrm{n}]=\mathrm{h}[\mathrm{n}] * \mathrm{x}[\mathrm{n}]+\mathrm{w}[\mathrm{n}]
$$

Where $\mathrm{h}=$ Impulse response of casual LTI system with $\mathrm{N}$-tap wireless channel

$\mathrm{x}=$ Transmitted vector

$\mathrm{w}=\mathrm{AWGN}$ noise vector

The frequency selective multipath channel is modeled as circular convolution [31] with a combination of CP and linear convolution. Therefore, the receiver vector is obtained after removing $\mathrm{CP}$, and serial to parallel conversion is performed and is given as

$$
y=H x+w=H A d+w=W_{D}^{H} \operatorname{diag}\left(W_{D} A d\right) F_{N} h+w
$$

Where $F_{N}=\left[\sqrt{D} W_{D}\right]_{:, 1: N}$ and $H \in C^{D x D}$ is the circular matrix. The estimated GFDM block $\hat{d}$ is obtained after performing the demodulation. The demodulation is dependent on the variation of the receiver targeted applications. The limitation of GFDM is a design of a low complex transceiver, a combination of mMIMOGFDM leads to large block size which makes the convolutional demodulation procedure makes it infeasible and synchronization error occurs. Because of these synchronization errors, training-based channel estimation is difficult.

\section{Results and Discussion}

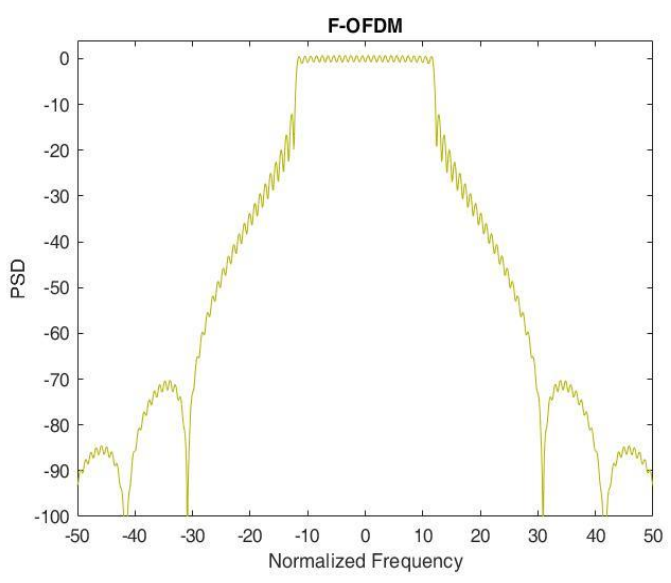

Figure 9: F-OFDM PSD 
The power spectral density (PSD) of F-OFDM concerning Normalized frequency where the number of subcarriers is 96 and subcarrier spacing frequency is $30 \mathrm{KHz}$ is shown in figure 9 .

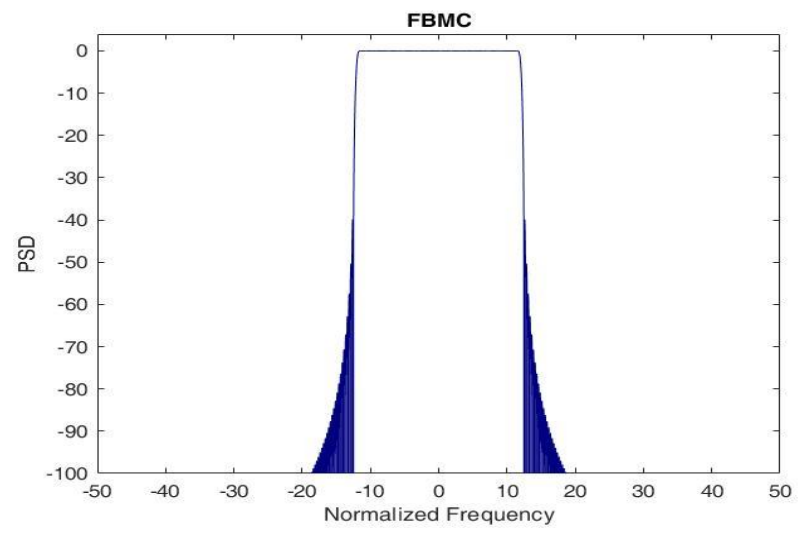

Figure 10: PSD of FBMC

PSD of FBMC concerning Normalized frequency where the number of subcarriers is 96 and subcarrier spacing frequency is $30 \mathrm{KHz}$ is shown in figure 10 .

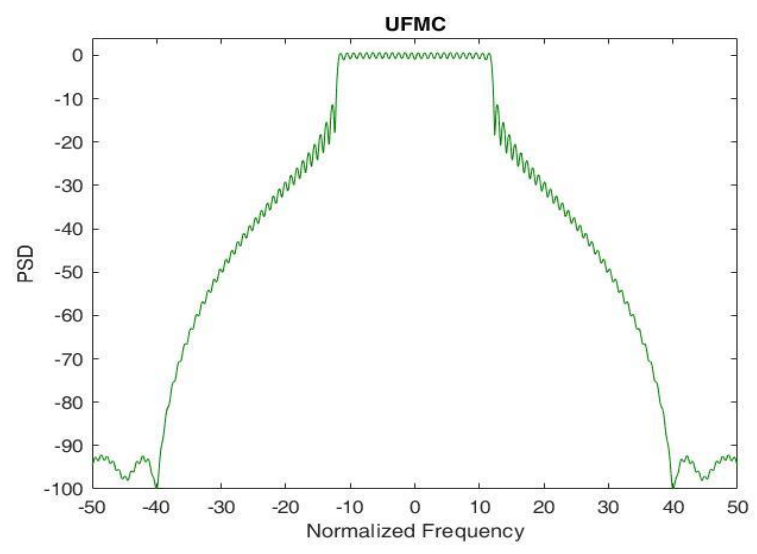

Figure 11: PSD of UFMC

The PSD of UFMC concerning Normalized frequency where the number of subcarriers is 96 and subcarrier spacing frequency is $30 \mathrm{KHz}$ is shown in figure 11.

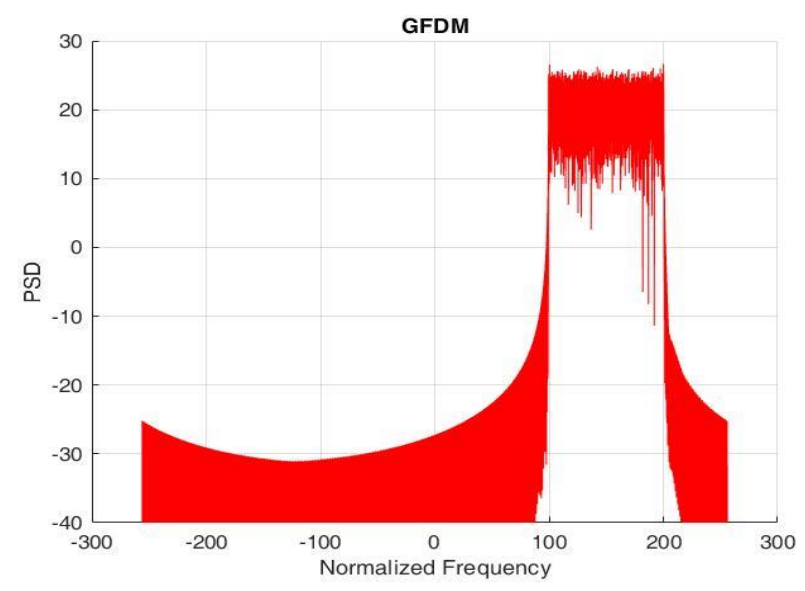

Figure 12: PSD of GFDM 
The PSD of GFDM concerning Normalized frequency where the number of subcarriers is 96, subcarrier spacing frequency is $30 \mathrm{KHz}$, and a number of blocks are 3 is shown in figure 12 .

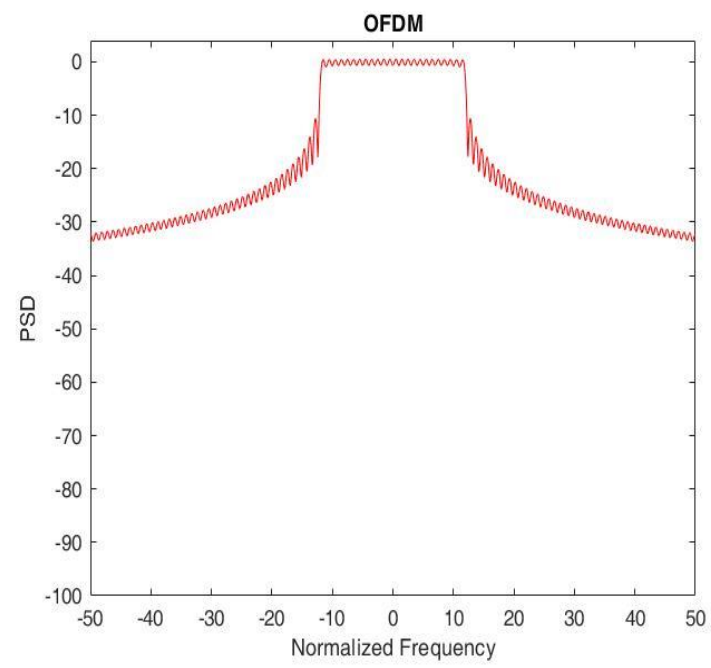

Figure 13: PSD of FBMC

PSD of FBMC concerning Normalized frequency where the number of subcarriers is 96 and subcarrier spacing frequency is $30 \mathrm{KHz}$ is shown in figure 13.

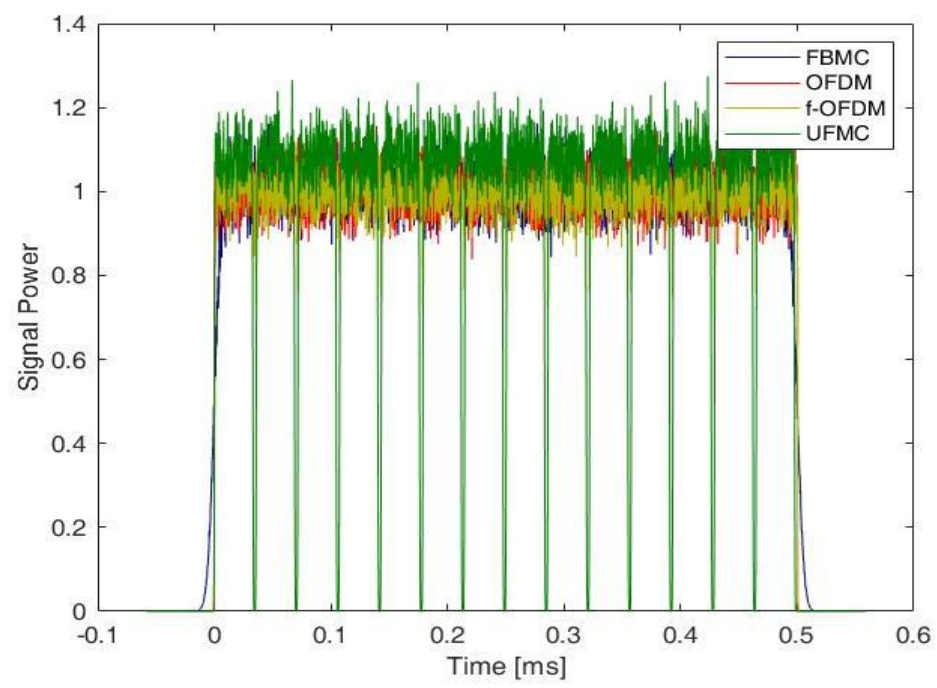

Figure 14: Signal Power of the candidate Waveforms.

The comparison of signal power of all candidate waveforms concerning time is shown in figure 14 where the number of subcarriers is 96 . 


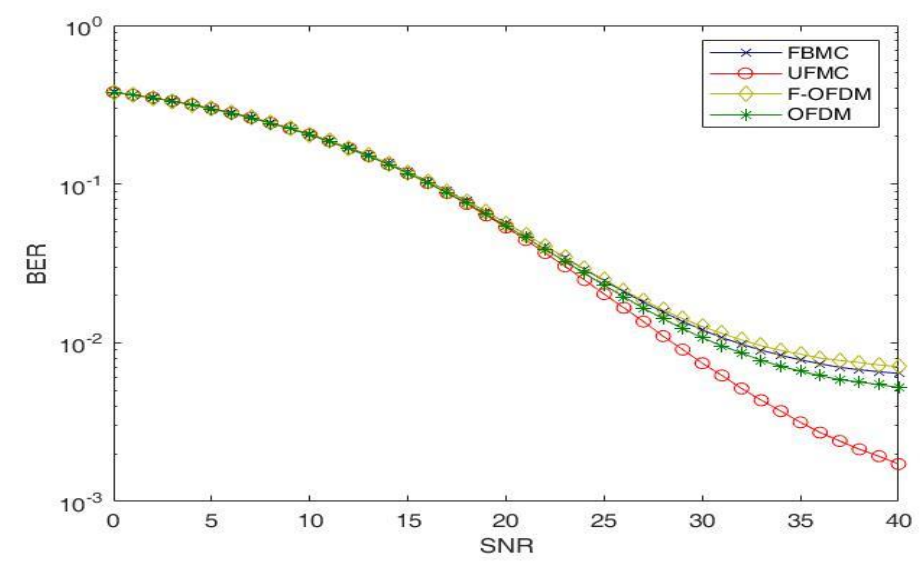

Figure 15: BER of the candidate Waveforms.

The comparison of a Bit Error rate of all candidate waveforms concerning SNR is shown in figure 15. The figure shows that as there is a rise in the SNR the BER of the UFMC reduces compared to other multi-carrier modulation techniques.

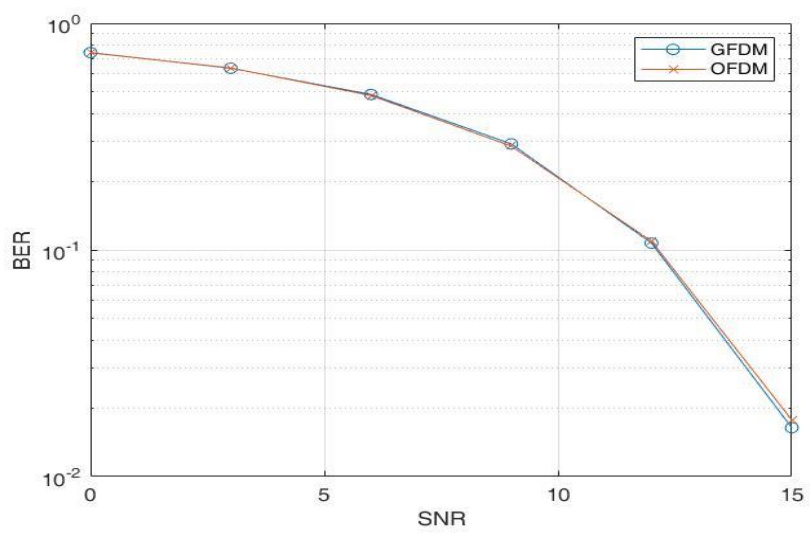

Figure 16: Bit Error Rate of the candidate Waveforms.

The comparison of a Bit Error rate of OFDM and GFDM concerning SNR is shown in figure 16. The figure shows that as the SNR value increases the BER of the GFDM reduces compared to OFDM.

4. Conclusion: There is a rapid increase in connectivity of the wireless system, almost an exponential rise in wireless data. The next generation $5 \mathrm{G}$ WCS is been a promising technology for an increase in high data rate, QoS, and connectivity. A new application such as IoT, IoV, and smart grids are supported under the 5G WCS. There are many promising candidate waveforms for 5G WCS such as FBMC, UFMC, FOFDM and GFDM, and many more. Compared to OFDM the other candidate waveforms are best suited for the 5G WCS. The PSD and BER performance of all multi-carrier modulation techniques is shown in this paper where a 16QAM modulation scheme is employed. As GFDM is a non-orthogonal multi-carrier modulation scheme its performance is compared only with the OFDM as it is mainly supported for the cognitive radio application. 


\section{Declaration:}

We the undersigned declare that this manuscript is original, has not been published before and is not currently being considered for publication elsewhere.

We confirm that the manuscript has been read and approved by all named authors and that there are no other persons who satisfied the criteria for authorship but are not listed. We further confirm that the order of authors listed in the manuscript has been approved by all of us.

We understand that the Corresponding Author is the sole contact for the Editorial process. He/she is responsible for communicating with the other authors about progress, submissions of revisions and final approval of proofs.

\section{References:}

[1] Akyildiz, Ian \& Nie, Shuai \& Lin, Shih-Chun \& Chandrasekaran, Manoj. (2016). 5G Roadmap: 10 Key Enabling Technologies. Computer Networks. 106. 10.1016/j.comnet.2016.06.010.

[2] F. Schaich, B. Sayrac, M. Schubert, H. Lin, K. Pedersen, M. Shaat, G. Wunder, and A. Georgakopoulos, "FANTASTIC-5G: 5G-PPP project on 5G air interface below $6 \mathrm{GHz}$," in European Conference on Network and Communications, 2015.

[3] L. Zhang, A. Ijaz, P. Xiao, and R. Tafazolli, “Multi-service system: An enabler of flexible 5G air interface,” IEEE Communications Magazine, vol. 55, no. 10, pp. 152-159, OCTOBER 2017.

[4] A. Ijaz, L. Zhang, M. Grau, A. Mohamed, S. Vural, A. U. Quddus, M. A. Imran, C. Foh, and R. Tafazolli, "Enabling Massive IoT in 5G and Beyond Systems: PHY Radio Frame Design Considerations,” IEEE Access, vol. 4, pp. 3322$3339,2016$.

[5] E. Dahlman, S. Parkvall, and J. Skold, 4G: LTE/LTE-Advanced for Mobile Broadband. Academic Press, 2011.

[6] L. Zhang, A. Ijaz, P. Xiao, and R. Tafazolli, "Subband filtered multicarrier systems for multi-service wireless communications," IEEE Transactions on Wireless Communications, vol. 16, no. 3, pp. 1893-1907, March 2017.

[7] Manolakis, Konstantinos \& Jungnickel, Volker \& Oberli, Christian \& Wild, Thorsten \& Braun, Volker \& Vucic, Nikola \& Castañeda Garcia, Mario. (2015). Cooperative Cellular Networks: Overcoming the Effects of Real-World Impairments. IEEE Vehicular Technology Magazine. 10.1109/MVT.2015.2446420.

[8] J. Abdoli, M. Jia, and J. Ma, "Filtered OFDM: A new waveform for future wireless systems," in IEEE Signal Processing Advances in Wireless Communications (SPAWC), June 2015, pp. 66-70.

[9] L. Zhang, P. Xiao, A. Zafar, A. ul Quddus, and R. Tafazolli, "FBMC system: An insight into doubly dispersive channel impact,” IEEE Transactions on Vehicular Technology, vol. PP, no. 99, pp. 1-14, 2016.

[10] B. Farhang-Boroujeny, “OFDM versus filter bank multicarrier,” IEEE Signal Processing Magazine, vol. 28, no. 3, pp. 92-112, May 2011.

[11] D. Chen, D. Qu, T. Jiang, and Y. He, "Prototype filter optimization to minimize stopband energy with NPR constraint for filter bank multicarrier modulation systems," IEEE Transactions on Signal Processing, vol. 61, no. 1, pp. 159-169, 2013.

[12] V. Vakilian, T. Wild, F. Schaich, S. Ten Brink, and J.-F. Frigon, "Universal-filtered multi-carrier technique for wireless systems beyond LTE,” in IEEE Globecom Workshops (GC Wkshps), 2013, pp. 223-228. 
[13] X. Wang, T. Wild, and F. Schaich, "Filter optimization for carrier frequency- and timing-offset in universal filtered multi-carrier systems,” in IEEE Vehicular Technology Conference (VTC Spring), 2015, pp. 1-6.

[14] N. Michailow, M. Matthe, I. Gaspar, A. Caldevilla, L. Mendes, A. Festag, and G. Fettweis, “Generalized frequency division multiplexing for 5th generation cellular networks," IEEE Transactions on Communications, vol. 62, no. 9, pp. 3045-3061, Sept 2014.

[15] G. Fettweis, M. Krondorf, and S. Bittner, "GFDM - generalized frequency division multiplexing," in IEEE Vehicular Technology Conference, April 2009, pp. 1-4.

[16] Matz, Gerald \& Schafhuber, Dieter \& Gröchenig, Karlheinz \& Hartmann, Manfred \& Hlawatsch, Franz. (2007). Analysis, Optimization, and Implementation of Low-Interference Wireless Multicarrier Systems. Wireless Communications, IEEE Transactions on. 6. 1921 - 1931. 10.1109/TWC.2007.360393.

[17] Jung, Peter \& Wunder, Gerhard. (2007). WSSUS Pulse Design Problem in Multicarrier Transmission. Communications, IEEE Transactions on. 55. 1918 - 1928. 10.1109/TCOMM.2007.906426.

[18] A. Sahin, I. Guvenc, and H. Arslan, "A survey on multicarrier communications: Prototype filters, lattice structures, and implementation aspects," IEEE Communications Surveys Tutorials, vol. 16, no. 3, pp. 1312-1338, December 2012. [19] R.Gerzaguet, D. Kténas, N. Cassiau, and J-B. Doré (CEA-LETI) "Comparative study of 5G waveform candidates for below 6GHz air interface" In Proceedings of the ETSI Workshop on Future Radio Technologies-Air Interface, Sophia Antipolis, France, 27-28 January 2016; pp. 1-9.

[20] X. Zhang, M. Jia, L. Chen, J. Ma, and J. Qiu, "Filtered-OFDM - Enabler for Flexible Waveform in the 5th Generation Cellular Networks," 2015 IEEE Global Communications Conference (GLOBECOM), San Diego, CA, 2015, pp. 1-6, DOI: 10.1109/GLOCOM.2015.7417854.

[21] Zhang, Lei \& Ijaz, Ayesha \& Xiao, Pei \& M. Molu, Mehdi \& Tafazolli, Rahim. (2017). Filtered OFDM Systems, Algorithms, and Performance Analysis for 5G and Beyond. IEEE Transactions on Communications. PP. 10.1109/TCOMM.2017.2771242.

[22] Zhang, Xi. (2009). Design of FIR Halfband Filters for Orthonormal Wavelets Using Remez Exchange Algorithm. Signal Processing Letters, IEEE. 16. 814 - 817. 10.1109/LSP.2009.2025829.

[23] Zhang, Lei \& Ijaz, Ayesha \& Xiao, Pei \& Quddus, Atta \& Tafazolli, Rahim. (2016). Single-rate and Multi-rate Multi-service Systems for Next Generation and Beyond Communications. 10.1109/PIMRC.2016.7794635.

[24] Haas, Ralf \& Belfiore, Jean-Claude. (1997). A Time-Frequency Well-localized Pulse for Multiple Carrier Transmission. Wireless Personal Communications. 5. 1-18. 10.1023/A:1008859809455.

[25] Nissel, Ronald \& Rupp, Markus. (2016). On pilot-symbol aided channel estimation in FBMC-OQAM. 3681-3685. 10.1109/ICASSP.2016.7472364.

[26] Bellanger, Maurice. (2001). Specification and design of prototype filter for filter bank based multicarrier transmission. Acoustics, Speech, and Signal Processing, IEEE International Conference on. 4. 2417-2420. 10.1109/ICASSP.2001.940488.

[27] Mirabbasi, S.. (2002). Design of prototype filter for near-perfect-reconstruction overlapped complex-modulated transmultiplexers. I-821 . 10.1109/ISCAS.2002.1009967.

[28] Geng, Suiyan \& Xiong, Xin \& Cheng, Linlin \& Zhao, Xiongwen \& Huang, Biao. (2015). UFMC system performance analysis for discrete narrow-band private networks. 10.1109/MAPE.2015.7510319.

[29] G. Fettweis and S. Alamouti, "5G: Personal mobile internet beyond what cellular did to telephony," in IEEE Communications Magazine, vol. 52, no. 2, pp. 140-145, February 2014, DOI: 10.1109/MCOM.2014.6736754. 
[30] N. Michailow, M. Matth'e, I. S. Gaspar, A. N. Caldevilla, L. L. Mendes, A. Festag, and G. Fettweis, “Generalized Frequency Division Multiplexing for 5th Generation Cellular Networks," IEEE Transactions on Communications, vol. 62, no. 9, pp. 3045-3061, 2014.

[31] D. Tse and P. Viswanath, Fundamentals of wireless communication. Cambridge university press, 2005. 


\section{Figures}

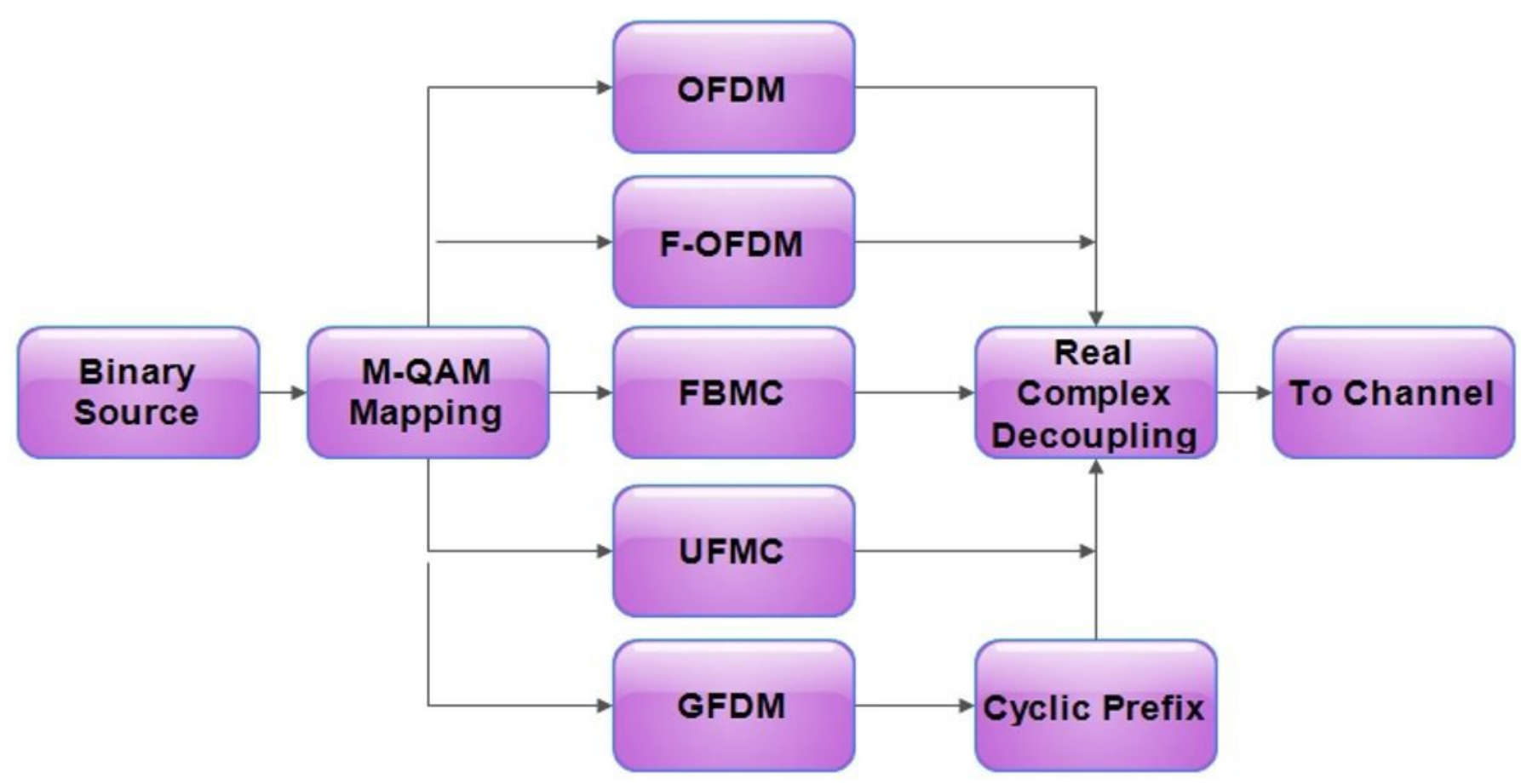

Figure 1

Waveform modulator block diagram.

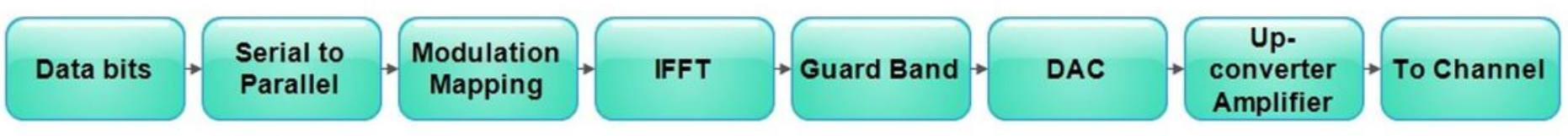

(a)

From
Channel $\rightarrow \begin{gathered}\text { Down- } \\ \text { converter } \\ \text { Amplifier }\end{gathered} \rightarrow$ ADC $\rightarrow \begin{aligned} & \text { Removal of } \\ & \text { Guard Band }\end{aligned} \rightarrow$ FFT Demapping $\rightarrow \begin{gathered}\text { Parallel to } \\ \text { Serial } \\ \text { Converter }\end{gathered} \rightarrow \begin{aligned} & \text { Received } \\ & \text { Data bits }\end{aligned}$

(b)

Figure 2

CP-OFDM transceiver block diagram a. Transmitter b. Receiver 


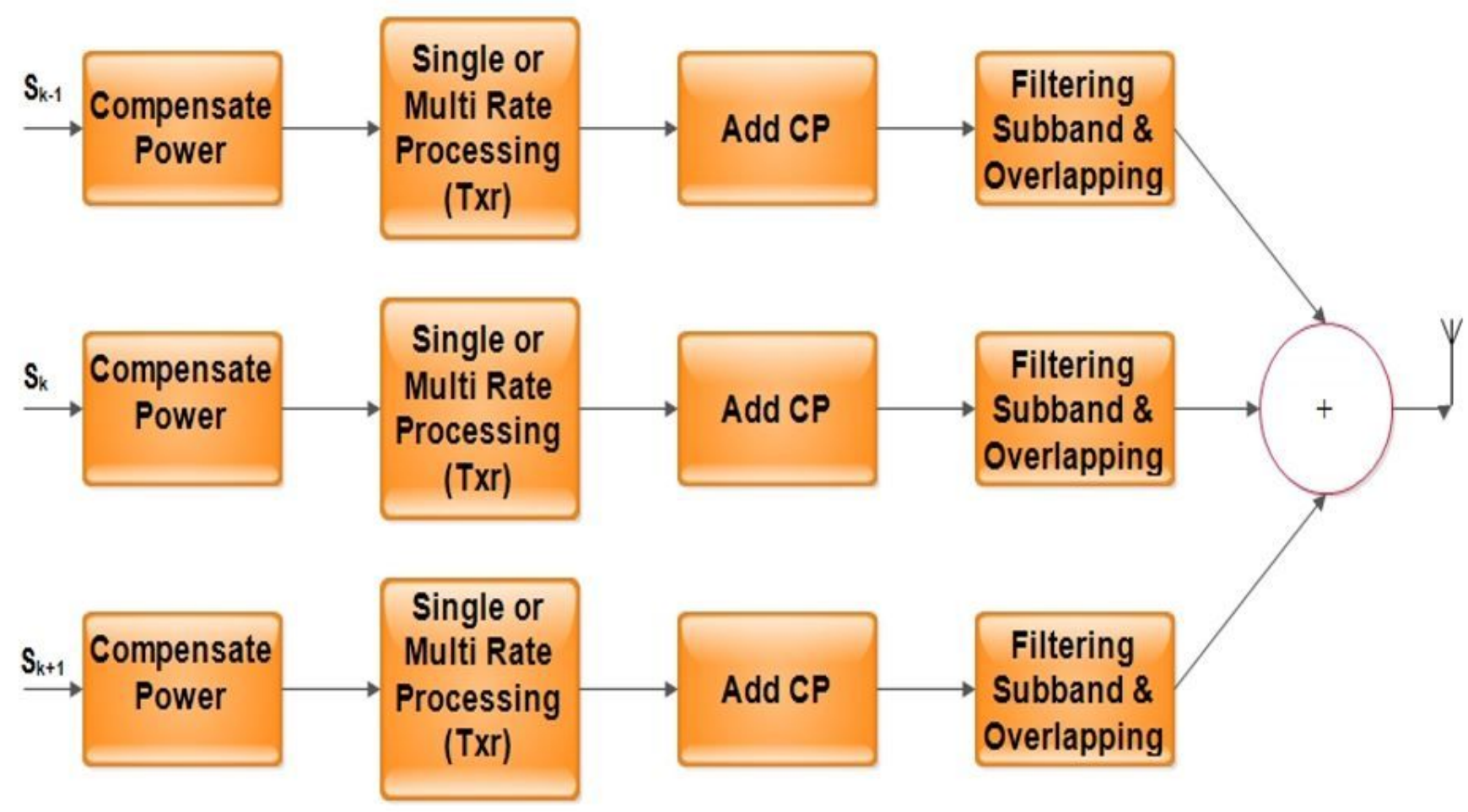

(a)

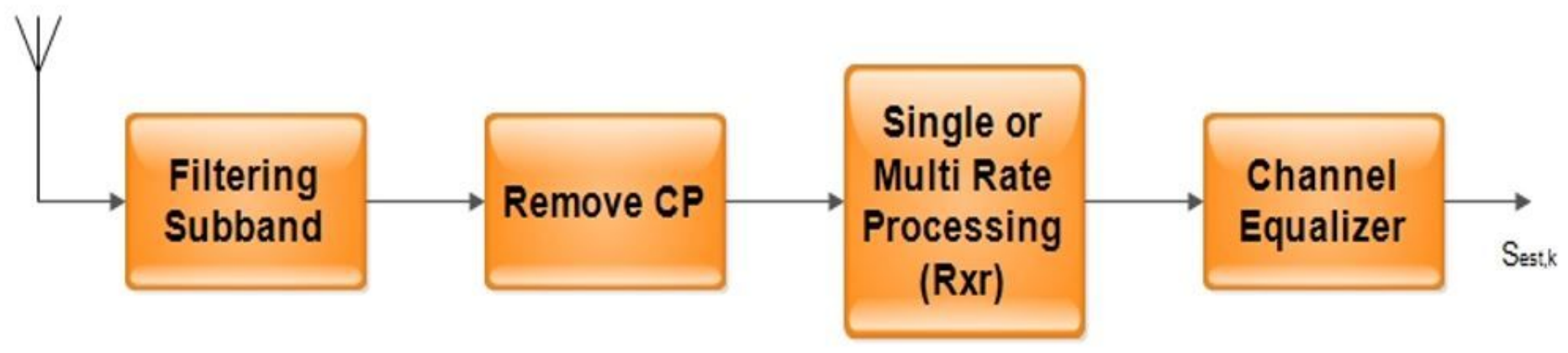

(b)

Figure 3

Transceiver of F-OFDM system model a. Transmitter b. Receiver.

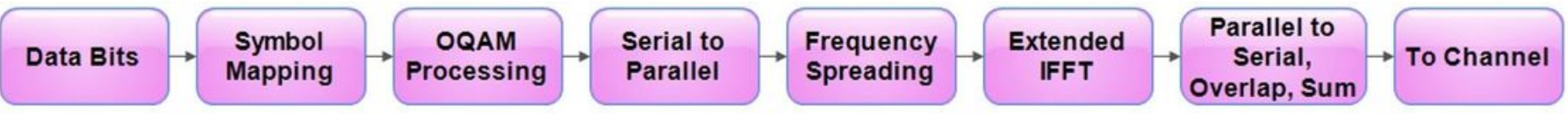

(a)

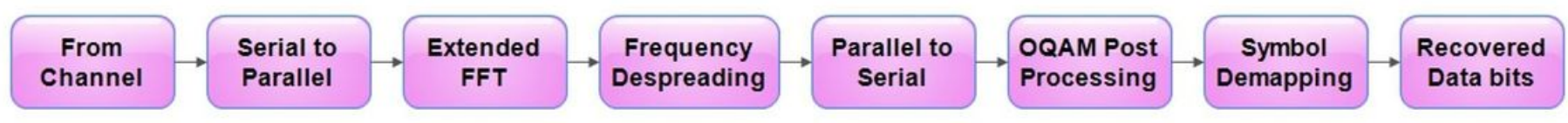

(b)

Figure 4 
Transceiver of FBMC a. Transmitter b. Receiver.

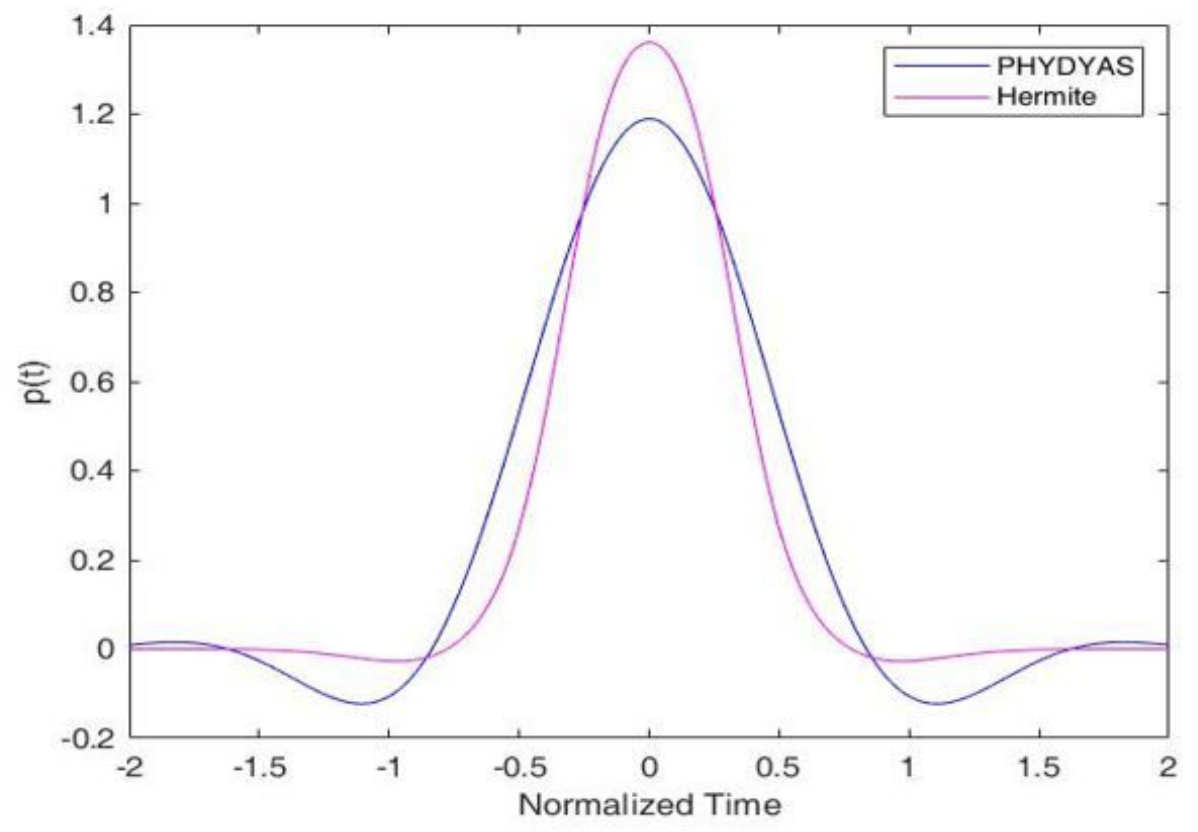

(a)

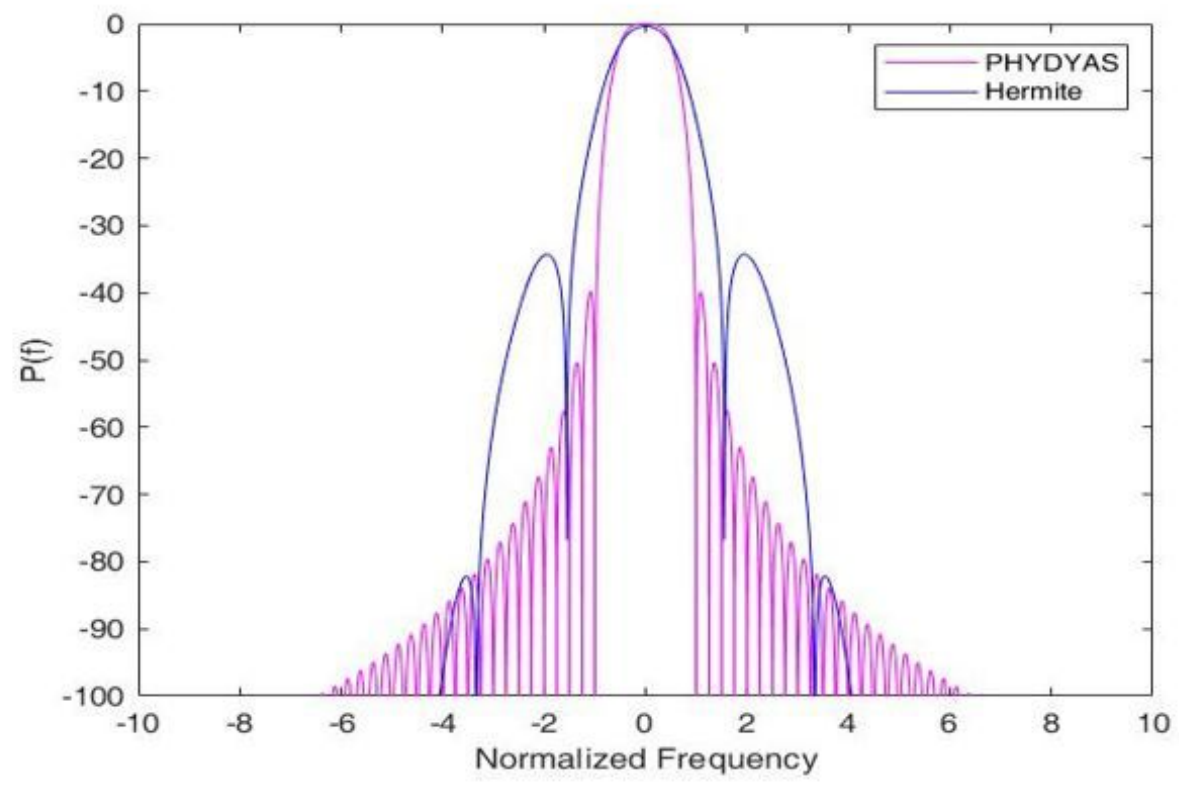

(b)

Figure 5

Prototype Filter for FBMC a. Time Domain Spacing b. Frequency Domain Spacing 


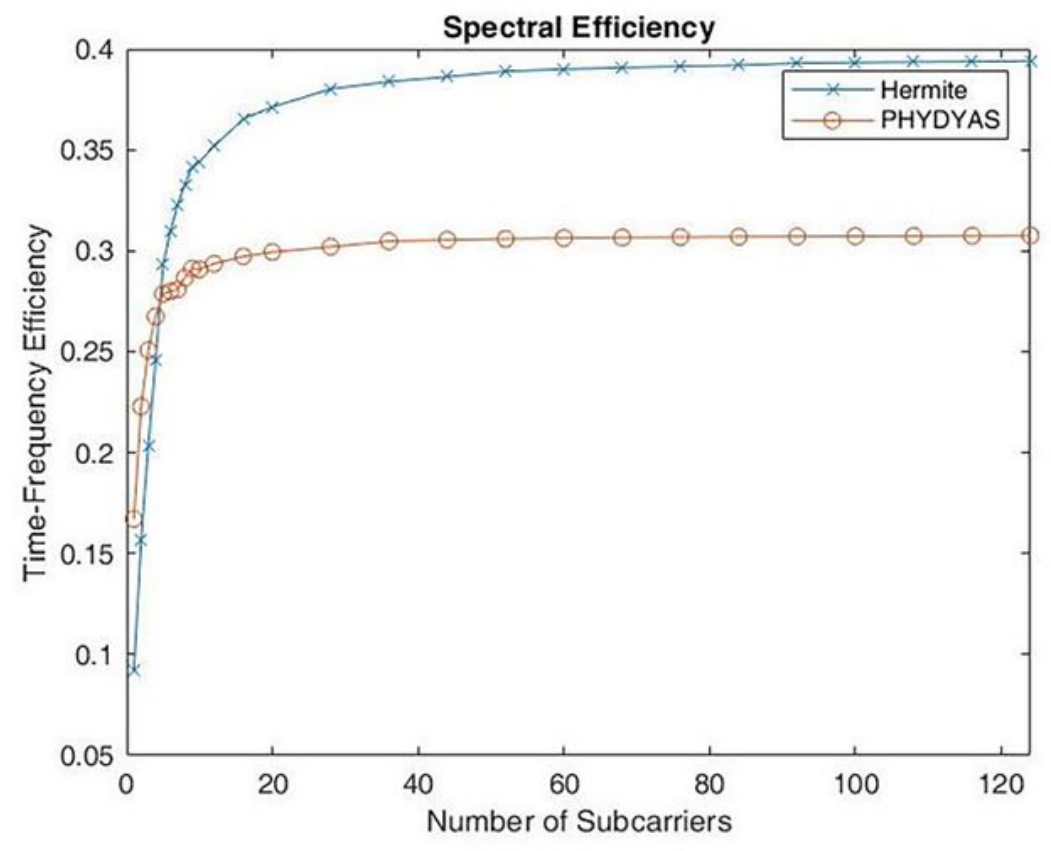

(a)

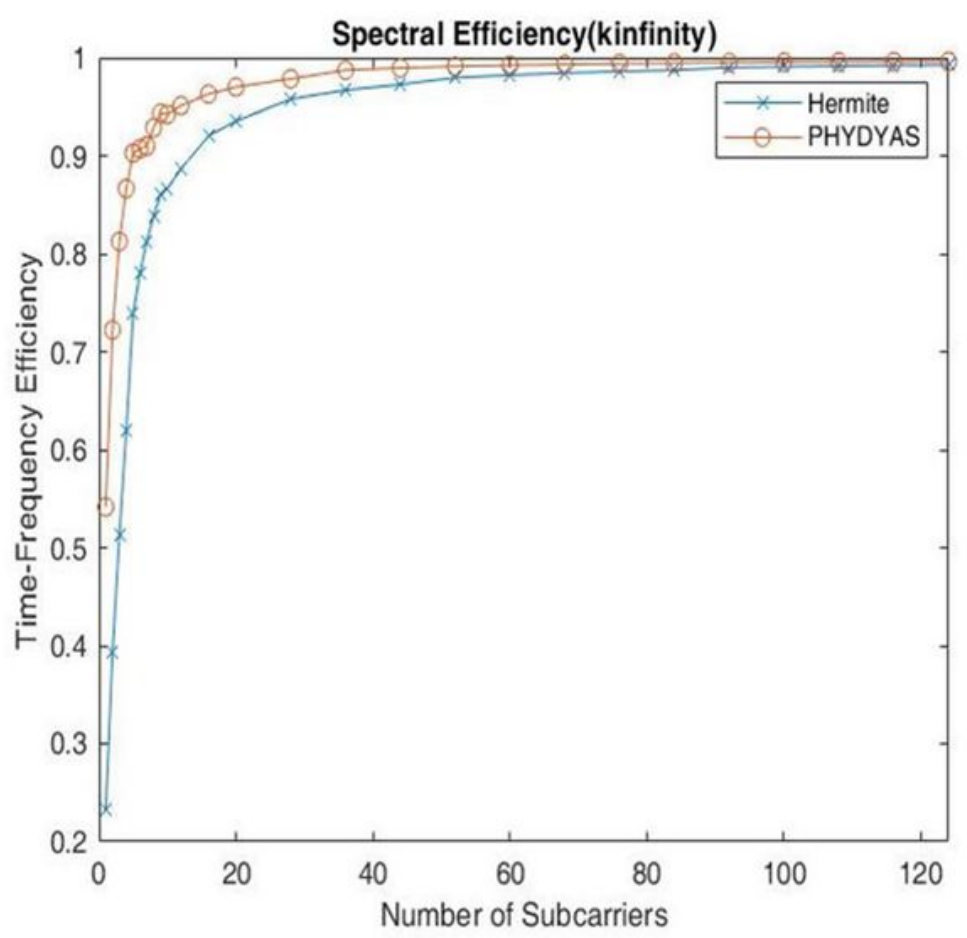

(b)

\section{Figure 6}

Spectral efficiency of Hermite and PHYDYAS Filter a. $K=$ minimum $b . K=$ Infinite 


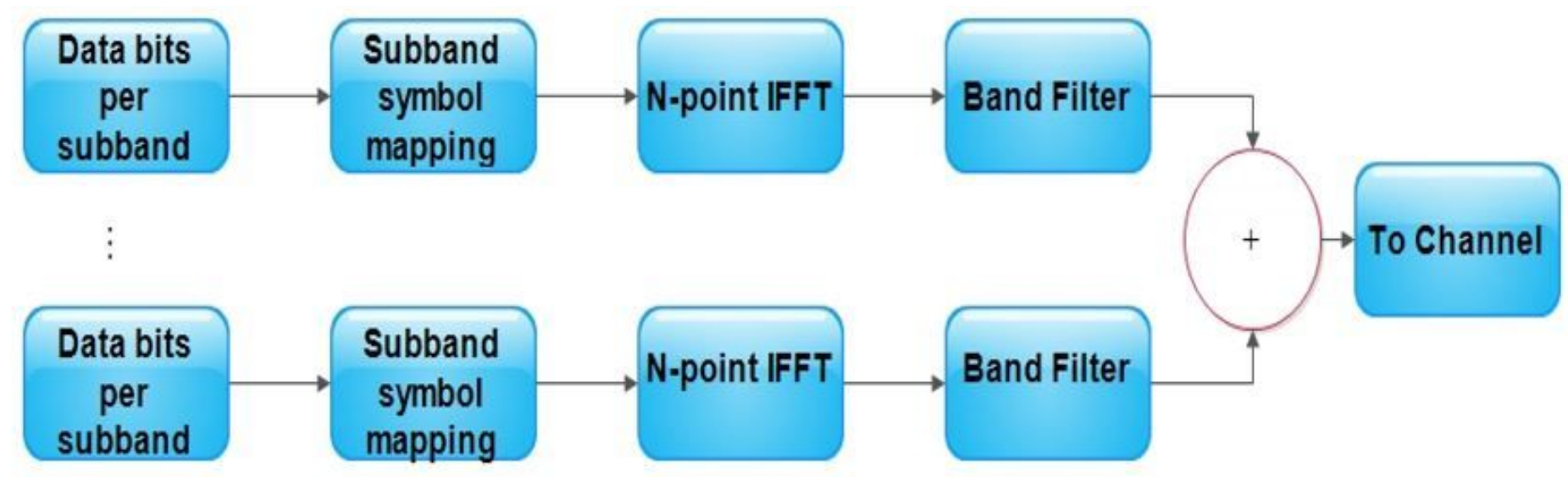

(a)

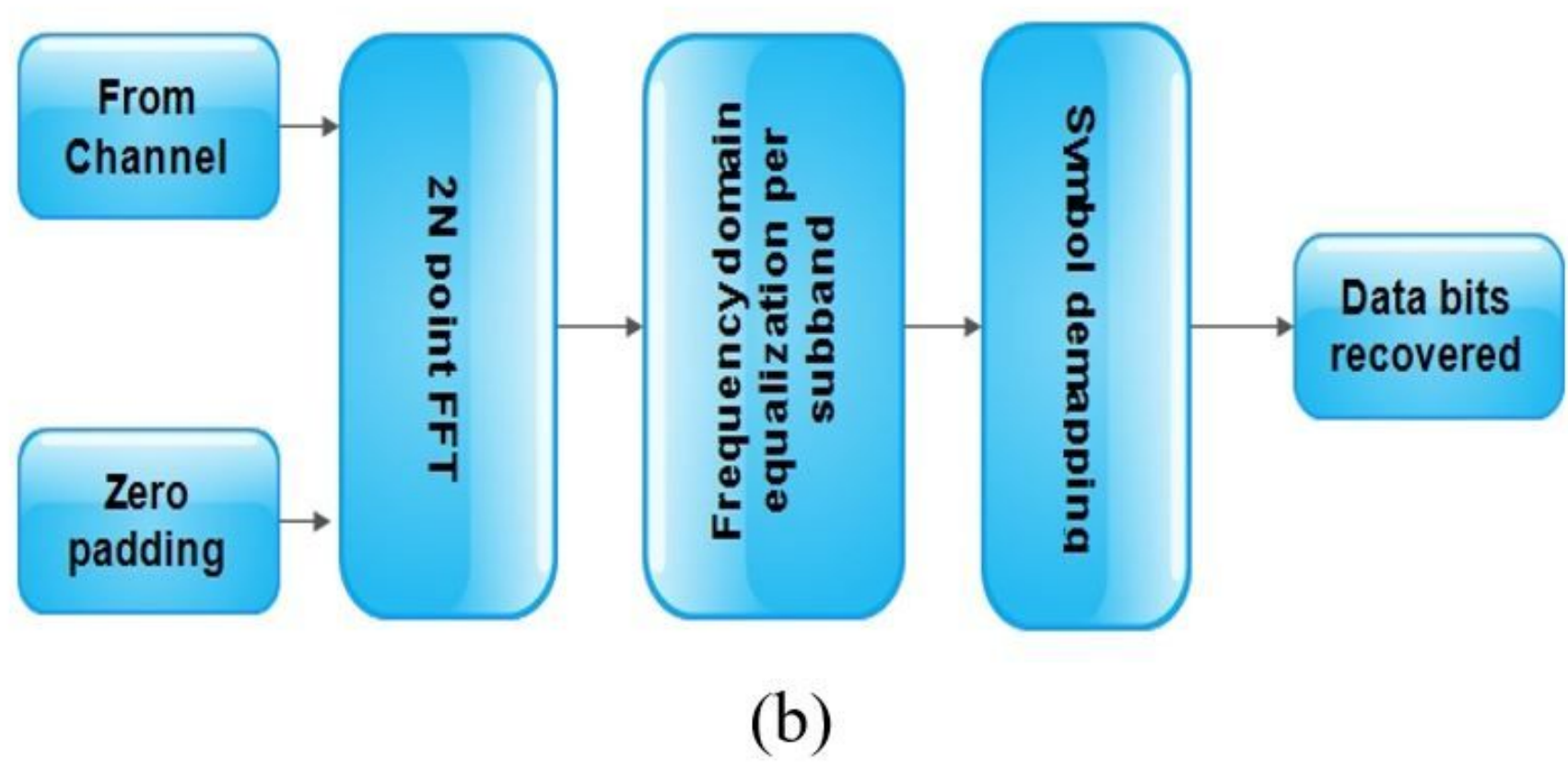

Figure 7

Transceiver of UFMC $a$. Transmitter b. Receiver. 


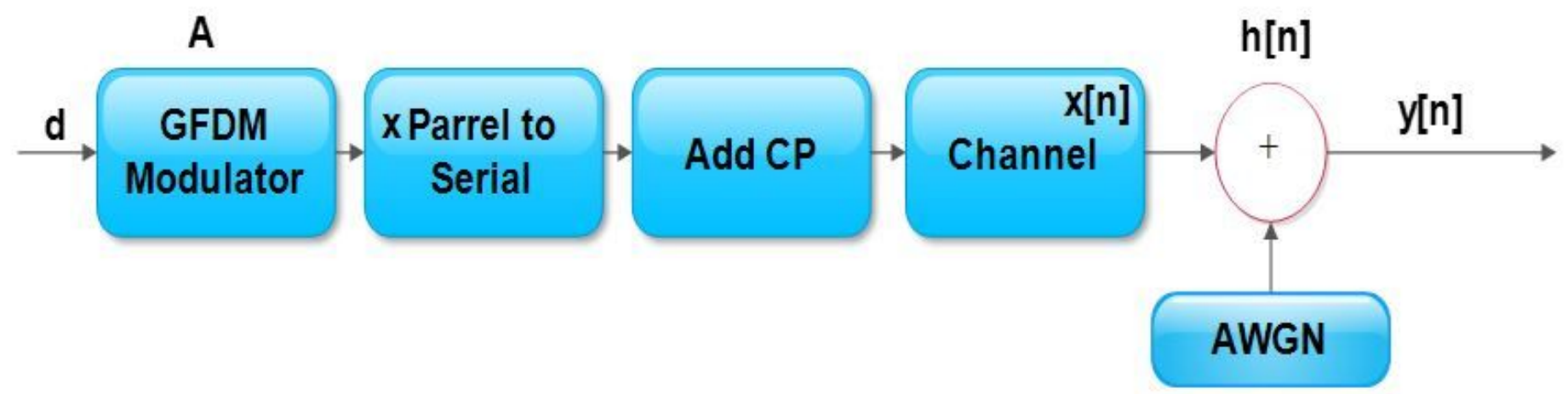

(a)

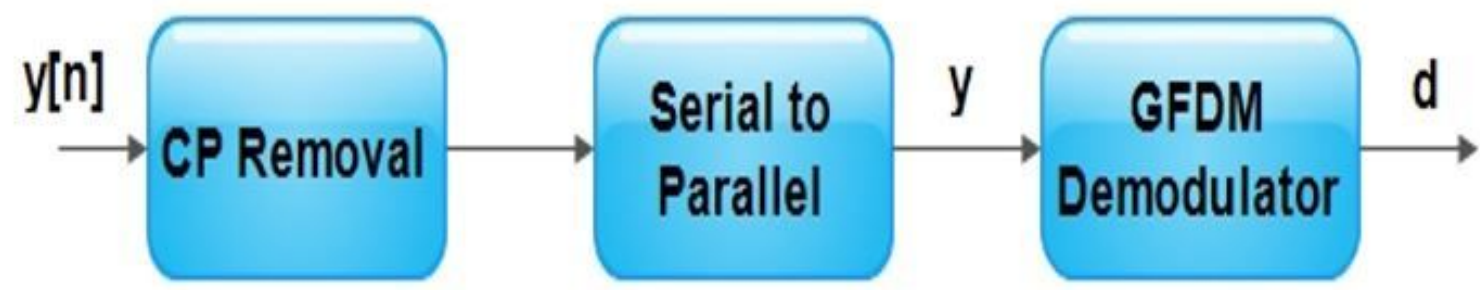

(b)

Figure 8

Transceiver of GFDM a. Transmitter b. Receiver 


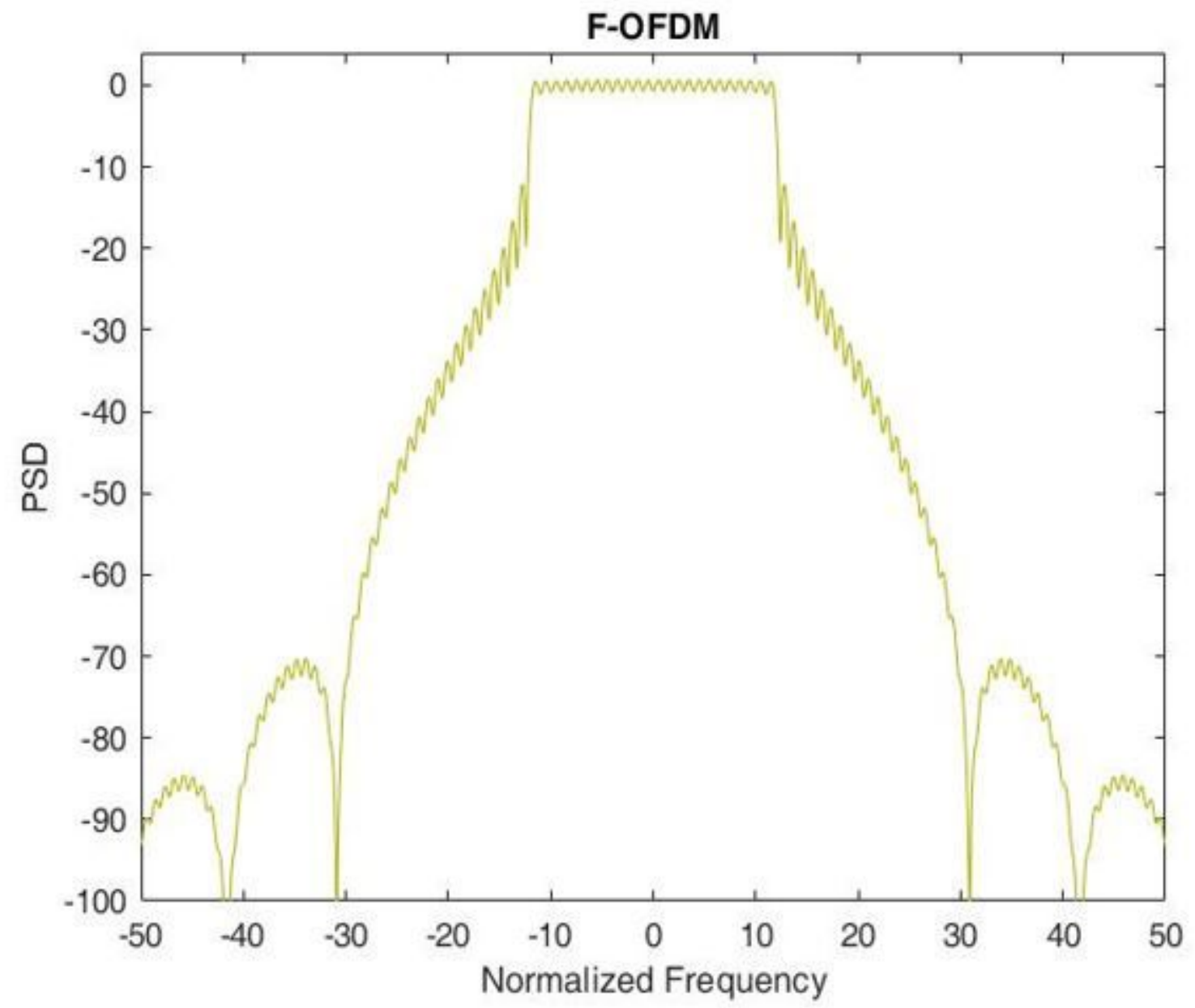

Figure 9

F-OFDM PSD 


\section{FBMC}

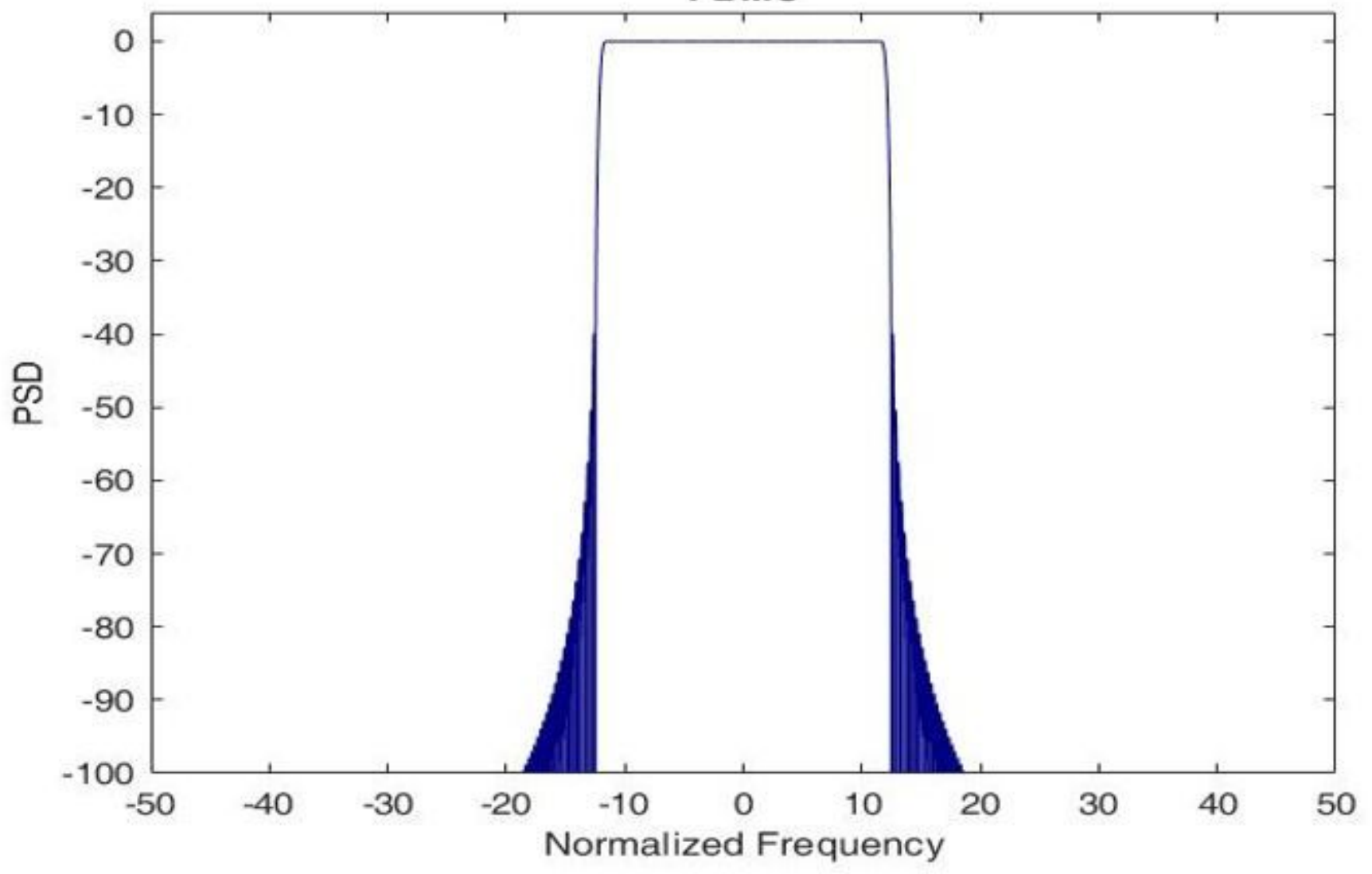

Figure 10

PSD of FBMC 


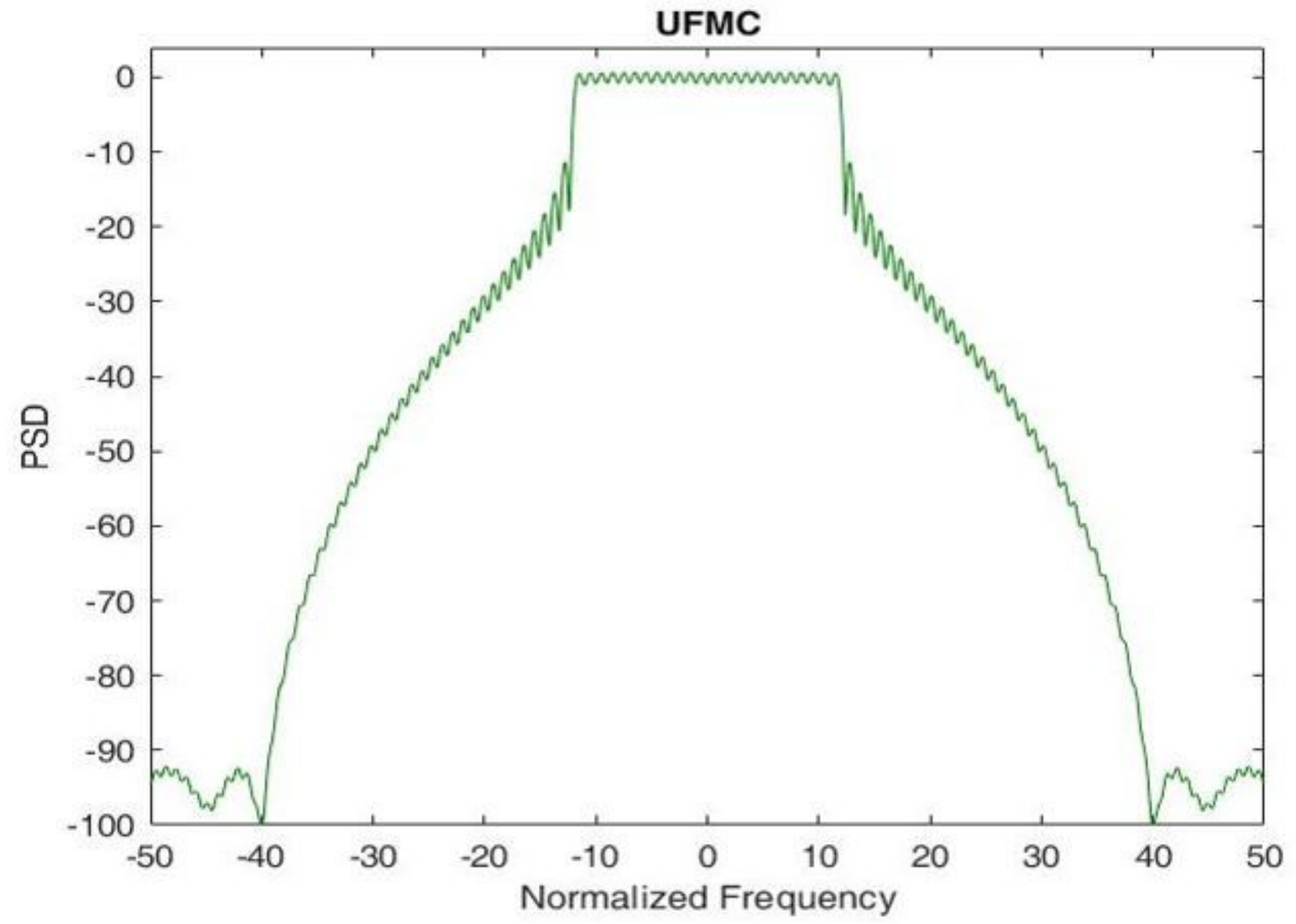

Figure 11

PSD of UFMC 


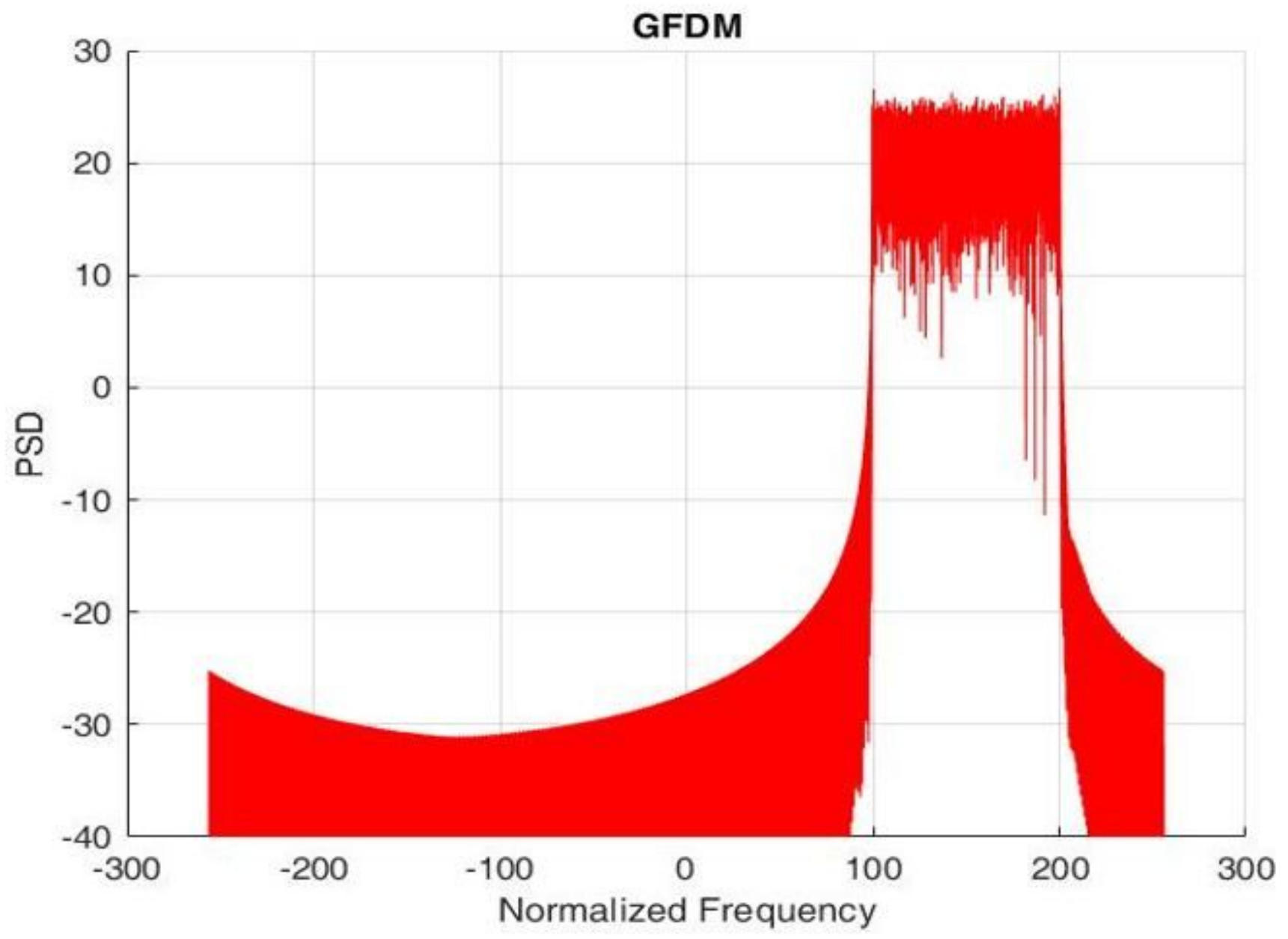

Figure 12

PSD of GFDM 


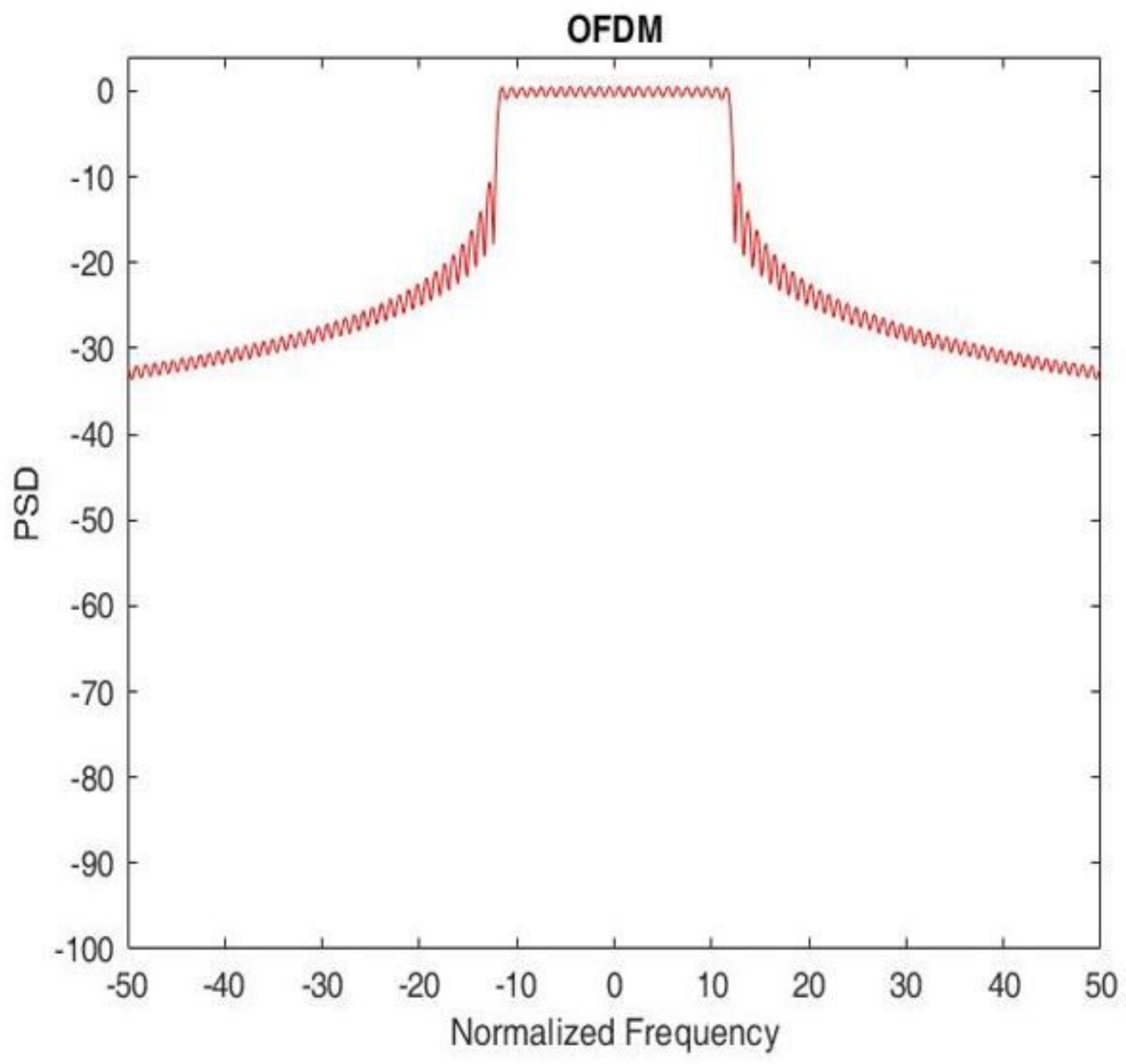

Figure 13

PSD of FBMC 


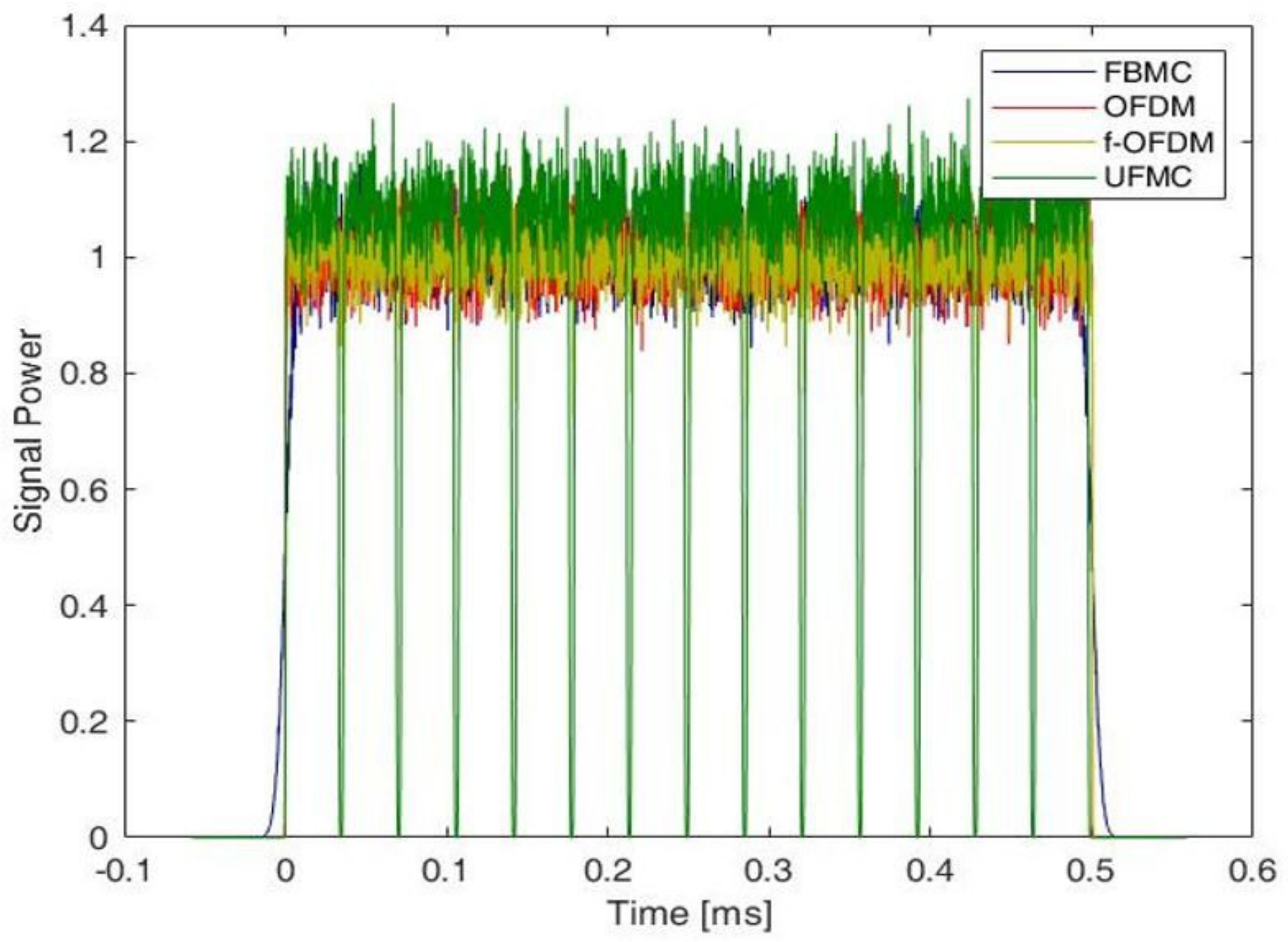

Figure 14

Signal Power of the candidate Waveforms. 


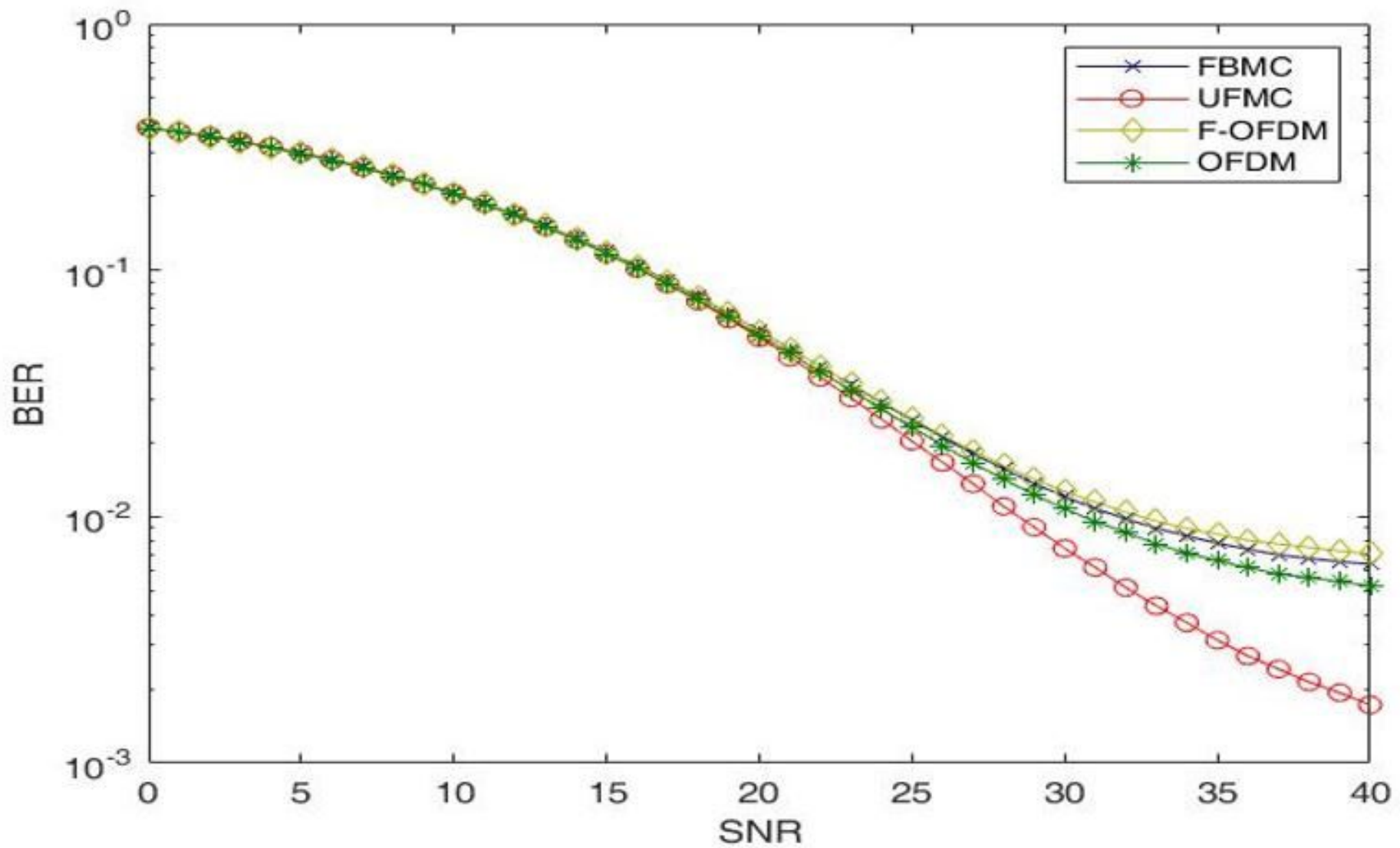

Figure 15

BER of the candidate Waveforms. 


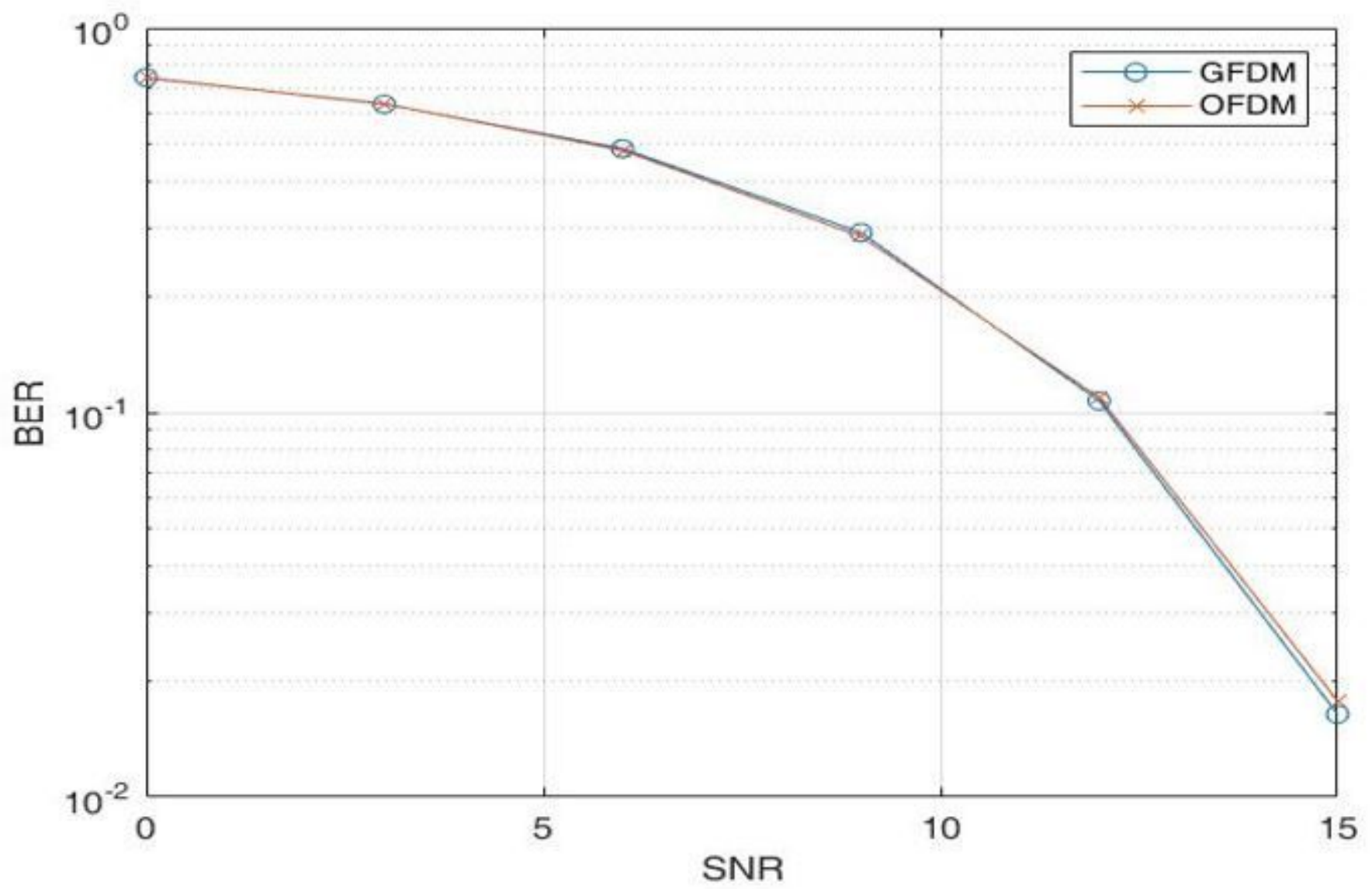

Figure 16

Bit Error Rate of the candidate Waveforms. 